\title{
Well-Balanced Positivity Preserving Cell-Vertex Central-Upwind Scheme for Shallow Water Flows
}

\author{
A. Beljadid ${ }^{\mathrm{a}, *}$, A. Mohammadian ${ }^{\mathrm{a}}$, A. Kurganov ${ }^{\mathrm{b}}$ \\ ${ }^{a}$ Department of Civil Engineering, University of Ottawa, 161 Louis Pasteur, Ottawa, ON, K1N6N5, \\ Canada \\ ${ }^{b}$ Mathematics Department, Tulane University, 6823 St. Charles Ave., New Orleans, LA, 70118, USA
}

\section{Abstract}

We develop a new second-order two-dimensional central-upwind scheme on cell-vertex grids for approximating solutions of the Saint-Venant system with source terms due to bottom topography. Central-upwind schemes are developed based on the information about the local speeds of wave propagation. Compared to the triangular central-upwind schemes, the proposed cell-vertex one has an advantage of using more cell interfaces which provide more information on the waves propagating in different directions. We propose a new piecewise linear approximation of the bottom topography and a novel non-oscillatory reconstruction in which the gradient of each variable is computed using a modified minmod-type method to ensure the stability of the scheme. A new technique is proposed for the correction of the water surface elevation which guarantees the positivity of the water depth. The well-balanced property of the proposed central-upwind scheme is ensured using a special discretization for the cell averages of the topography source terms. The proposed scheme is tested on a number of numerical examples, among which we consider steady-state solutions with almost dry areas and their perturbations and solutions with rapidly varying flows over discontinuous bottom topography. Our numerical experiments confirm stability, well-balanced, positivity preserving properties and second-order accuracy of the proposed method. This scheme can be applied to shallow water models when the bed topography is discontinuous and/or highly oscillatory, and on complicated domains where the use of unstructured grids is advantageous. 
Keywords: Shallow water equations, central-upwind schemes, finite-volume methods, cell-vertex grids, well-balanced schemes, positivity preserving property

\section{Introduction}

This paper focuses on development of modern numerical methods for the two-dimensional (2-D) Saint-Venant system of shallow water equations (SWEs):

$$
\left\{\begin{array}{l}
h_{t}+(h u)_{x}+(h v)_{y}=0 \\
(h u)_{t}+\left(h u^{2}+\frac{g}{2} h^{2}\right)_{x}+(h u v)_{y}=-g h B_{x} \\
(h v)_{t}+(h u v)_{x}+\left(h v^{2}+\frac{g}{2} h^{2}\right)_{y}=-g h B_{y} .
\end{array}\right.
$$

Here, $h$ is the water depth, $(u, v)^{T}$ is the velocity field, the function $B(x, y)$ represents the bottom elevation, and $g$ is the acceleration due to gravity.

Many upwind (see, e.g., $[1,2,5,9,10,18,22,31,33,37])$ and central (see, e.g., [6, 8, 15, $23,28,40,41,45])$ schemes for the shallow water system (1), which is a hyperbolic system of conservation (if $B_{x} \equiv B_{y} \equiv 0$ ) or balance (if $B$ is not a constant) laws, have been proposed in the past two decades. Roughly speaking, the main difference between upwind and central schemes is that upwind schemes use characteristic information and utilize (approximate) Riemann problem solvers to determine nonlinear wave propagation, while central schemes are based on averaging over the waves without using their detailed structures.

Riemann-problem-solver-free central schemes have become a very popular tool for hyperbolic systems of conservation and balance laws after the pioneer work of Nessyahu and

*Corresponding author

Email address: beljadid@mit.edu. Present address: Massachusetts Institute of Technology, 77 Massachusetts Avenue, Cambridge, Massachusetts 02139, USA. (A. Beljadid) 
Tadmor, [38], where a second-order, shock-capturing, finite-volume central scheme on a staggered grid was proposed. Since 1990, several higher-order and multidimensional extensions and generalizations of staggered central schemes have been introduced (see, e.g., [40] and references therein). However, staggered central schemes may not provide a satisfactory resolution when small time steps are enforced by stability restrictions, which may occur, for example, in the application of these schemes to convection-diffusion problems as observed in Kurganov and Tadmor [30]. These disadvantages are caused by the accumulation of numerical dissipation. Staggered central schemes can be improved by using some characteristic information on local speeds of propagation. This leads to a class of central-upwind schemes developed by Kurganov et al. in [25, 26, 27, 30]. The central-upwind schemes are based on (one-sided) local speeds which represent the extreme eigenvalues of the system. The use of these techniques makes the central-upwind schemes less dissipative compared to the staggered central schemes, and at the same time being Riemann-problem-solver-free methods they retain the major advantage of central schemes - simplicity. The central-upwind schemes have been successfully applied to a variety of problems including several shallow water models $[4,6,12,13,23,24,28,29,32]$.

SWEs and related models are of great interest for many atmospheric and oceanic applications as well as for modeling flows in the rivers and coastal areas. To be able to accurately model realistic situations, one has to develop numerical methods on unstructured grids due to their flexibility to represent irregular domains and convenience of local mesh refinement. There are two main proprieties a good numerical method for SWEs should satisfy. The first one is called a well-balanced property: the scheme should exactly preserve "lake at rest" steady-state solutions. The second property is positivity preserving: the method should guarantee positivity of the computed values of the water depth in each point of the domain at all times.

The main widely used unstructured finite-volume methods are cell-centered (CCFVM) 
and cell-vertex (CVFVM) ones. The cell-vertex methods are sometimes referred to as nodecentered, mesh-vertex or vertex-centered methods. For the CCFVM, the cells are the triangles of the primary mesh. For CVFVM the cells are the dual of the primary mesh as explained in the next section. For a detailed discussion on the two methods we refer the reader to $[3,34,36]$.

Diskin et al. [17] have compared the node-centered and cell-centered schemes for finitevolume discretization of Poisson's equation as a model with viscous fluxes. They have tested structured and unstructured grids based on both triangular and quadrilateral computational cells with randomly perturbed grid points. The authors found that the node-centered finite-volume methods typically outperform the cell-centered ones in terms of accuracy and convergence when the same number of degrees of freedom is used.

Nikolos and Delis [39] proposed a cell-vertex upwind scheme for shallow water flows with wet/dry fronts over complex bottom topography. The authors used the Roe method to compute numerical fluxes and the time evolution of their scheme was carried out by an explicit four-stage Runge-Kutta method. Delis et al. [16] have recently performed an extensive comparison between node-centered and cell-centered upwind finite-volume methods for the 2-D SWEs with different source terms on unstructured grids. They studied the performance, robustness and defectiveness of the two methods by comparing numerical results with both analytical solutions and experimental and field data. In the analyses, the authors used different structures of computational grids and the comparisons were performed for all conservative variables using different norms. They found that the CVFVM leads to identical convergence behavior for grids with various qualities (in terms of orientation and distortion) while in the CCFVM, the results are influenced by the grid quality. The reason is that the cells in the CVFVM are constructed in a way that leads to more spatial uniformity than the CCFVM. The authors concluded in their analyses that the CCFVM require more attention in order to obtain an appropriate correction in the construction of the extrapolated primi- 
tive variables of the system. Without adequate correction, the points where the numerical fluxes are evaluated do not correspond to the flux vectors obtained by extrapolations. The quality of the results of the CVFVM are less affected by the grid geometry. In addition to the advantages already mentioned, the CVFVM present an advantage compared to the CCFVM for the treatment of the boundary conditions since in the case of the CVFVM the control volume centers can be located on the boundary of the computational domain.

To the best of our knowledge, no cell-vertex central-upwind scheme for shallow water flows or hyperbolic systems of conservation laws have been proposed in the literature. Bryson et al. [6] have proposed a central-upwind scheme on triangular grids for the SaintVenant system of SWEs with possibly discontinuous bottom topography. The authors have showed that their method is well-balanced and positivity preserving, and demonstrated high resolution and robustness of the method. In this paper, we introduce a new well-balanced positivity preserving central-upwind scheme on cell-vertex grids (described in Section 2.1) for the 2-D SWEs with variable topography.

The paper is organized as follows. In Section 2, we present the new cell-vertex semidiscrete central-upwind scheme for the SWEs (1). In Section 3, we propose a positivity preserving reconstruction for water surface elevation. The well-balanced discretization of the source term is developed in Section 4. The positivity preserving property of the proposed scheme is proved in Section 5. In Section 6, we demonstrate the high resolution and robustness of the proposed method by testing it on a variety of numerical experiments. The final Section 7 contains concluding remarks.

\section{The Cell-Vertex Central-Upwind Scheme}

In this section, we focus on the derivation of the proposed cell-vertex central-upwind scheme. First the cell-vertex unstructured grid and the notations used in this paper are described in Section 2.1. Then, we develop the central-upwind method over cell-vertex grids 
for the SWEs (1), which can be rewritten using the vector of variables $\boldsymbol{U}:=(w, p, q)^{T}$ as

$$
\boldsymbol{U}_{t}+\boldsymbol{F}(\boldsymbol{U}, B)_{x}+\boldsymbol{G}(\boldsymbol{U}, B)_{y}=\boldsymbol{S}(\boldsymbol{U}, B)
$$

with

$$
\begin{aligned}
& \boldsymbol{F}(\boldsymbol{U}, B)=\left(p, \frac{p^{2}}{w-B}+\frac{g}{2}(w-B)^{2}, \frac{p q}{w-B}\right)^{T} \\
& \boldsymbol{G}(\boldsymbol{U}, B)=\left(q, \frac{p q}{w-B}, \frac{q^{2}}{w-B}+\frac{g}{2}(w-B)^{2}\right)^{T} \\
& \boldsymbol{S}(\boldsymbol{U}, B)=\left(0,-g(w-B) B_{x},-g(w-B) B_{y}\right)^{T}
\end{aligned}
$$

where $w:=h+B$ represents the water surface elevation and $p:=h u$ and $q:=h v$ denote the discharges in the $x$ - and $y$-directions, respectively.

\subsection{Cell-Vertex Grid and Notations}

Unstructured cell-vertex grids are obtained using a triangular discretization of the global domain $\mathcal{D}$ : The finite-volume cells, denoted by $M_{j}$, are centered around the vertices as shown in Figure 1. There are various methods to define the dual grid. The control volume around each node can be defined by connecting either the barycenters [39] or centroids [21] of the surrounding triangles of the node. These points can be connected either directly or with the midpoints of the edges that meet the node. In this paper, the boundary $\partial M_{j}$ of the cell $M_{j}$ around each internal triangulation vertex $P_{j}$ is defined by connecting directly the centers of mass of the surrounding triangles that have $P_{j}$ as a common vertex. The water surface elevation $w$ and the discharges $p$ and $q$ are then represented by the corresponding cell averages over the cells $M_{j}$ of size $\left|M_{j}\right|$ with the centers of mass denoted by $G_{j} \equiv\left(x_{j}, y_{j}\right)$.

We assume that the discretization $\mathcal{D}=\bigcup_{j=1}^{N} M_{j}$ consists of $N$ non-overlapping cells $(N$ is equal to the number of nodes of the initial triangular grid). To obtain the proposed semidiscrete scheme, we introduce the following notations for the cell $M_{j}$ and its neighboring 


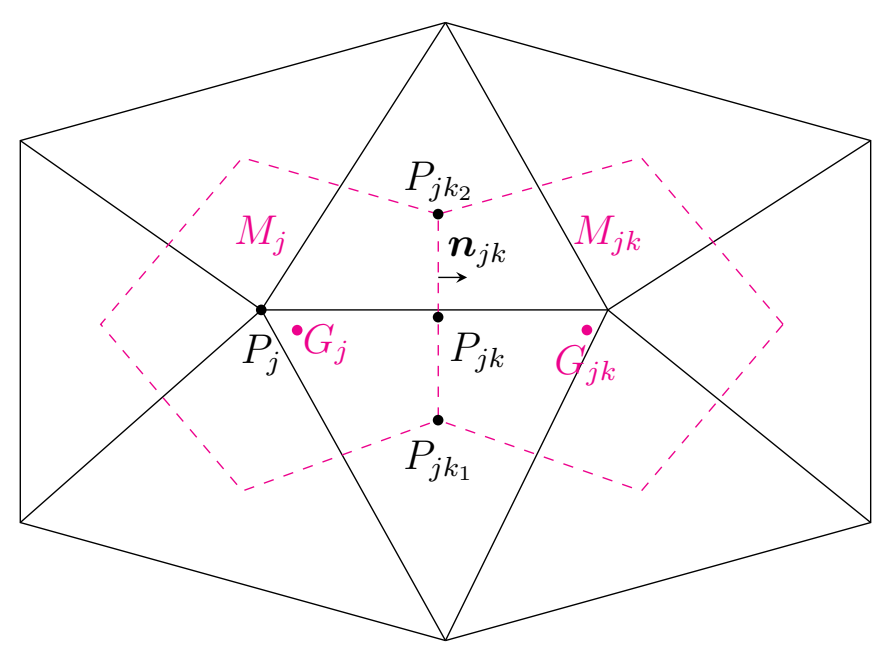

Figure 1: Sample of the cell-vertex. Solid lines represent the primary triangular grids and the dashed lines show the computational polygonal cells.

cells (notice that these notations are not global). For each cell $M_{j}$ we denote by $m_{j}$ the number of its cell sides and by $M_{j 1}, M_{j 2}, \ldots, M_{j m_{j}}$ the neighboring cells that share with $M_{j}$ a common side $\left(\partial M_{j}\right)_{1},\left(\partial M_{j}\right)_{2}, \ldots,\left(\partial M_{j}\right)_{m_{j}}$, respectively. The side length and the outward unit normal vectors of the cell-interfaces are denoted by $\ell_{j k}$ and $n_{j k}:=\left(\cos \theta_{j k}, \sin \theta_{j k}\right)^{T}$, respectively, where $\theta_{j k}$ is the angle of the unit normal vector $n_{j k}$ with the $x$-axis. The midpoint of the $k$ th edge of $M_{j}$ is denoted by $P_{j k}$ and the end points of this edge are denoted by $P_{j k_{s}}$, where $s=1,2$.

Note that the vertices of the cell $M_{j}$ may be denoted by $P_{j k_{1}}$, which is the starting point of the interface $k$ of this cell and for $k<m_{j}, P_{j k_{2}}=P_{j(k+1)_{1}}$. An anticlockwise orientation is used to define the start and end points of an interface. Similar indexing is used for other variables. For example, $w_{j k_{1}}, h_{j k_{1}}$ and $B_{j k_{1}}$ stand for the water surface elevation, water depth and the bottom elevation, respectively, at the vertex $P_{j k_{1}}$. Finally, $t^{n}$ denotes the $n$th time level.

Remark 1. It should be pointed out that although the initial grid is assumed to be triangular, the proposed cell-vertex method can be based on a quadrilateral or another polygonal unstructured initial grid. This leads to more flexibility of the cell-vertex methods 
compared to schemes that are restricted to triangular grids only.

\subsection{The Semi-Discrete Form of the Scheme}

The semi-discrete central-upwind scheme on cell-vertex grids can be derived following the procedure developed in [27] and [6] for triangular grids. It can be shown that the resulting scheme is

$$
\begin{aligned}
\frac{d \overline{\boldsymbol{U}}_{j}}{d t}= & -\frac{1}{\left|M_{j}\right|} \sum_{k=1}^{m_{j}} \frac{\ell_{j k} \cos \left(\theta_{j k}\right)}{a_{j k}^{\text {in }}+a_{j k}^{\text {out }}}\left[a_{j k}^{\text {in }} \boldsymbol{F}\left(\boldsymbol{U}_{j k}\left(P_{j k}\right), B_{j k}\right)+a_{j k}^{\text {out }} \boldsymbol{F}\left(\boldsymbol{U}_{j}\left(P_{j k}\right), B_{j k}\right)\right] \\
& -\frac{1}{\left|M_{j}\right|} \sum_{k=1}^{m_{j}} \frac{\ell_{j k} \sin \left(\theta_{j k}\right)}{a_{j k}^{\text {in }}+a_{j k}^{\text {out }}}\left[a_{j k}^{\text {in }} \boldsymbol{G}\left(\boldsymbol{U}_{j k}\left(P_{j k}\right), B_{j k}\right)+a_{j k}^{\text {out }} \boldsymbol{G}\left(\boldsymbol{U}_{j}\left(P_{j k}\right), B_{j k}\right)\right] \\
& +\frac{1}{\left|M_{j}\right|} \sum_{k=1}^{m_{j}} \ell_{j k} \frac{a_{j k}^{\text {in }} a_{j k}^{\text {out }}}{a_{j k}^{\text {in }}+a_{j k}^{\text {out }}}\left[\boldsymbol{U}_{j k}\left(P_{j k}\right)-\boldsymbol{U}_{j}\left(P_{j k}\right)\right]+\overline{\boldsymbol{S}}_{j},
\end{aligned}
$$

where $\overline{\boldsymbol{U}}_{j} \approx \frac{1}{\left|M_{j}\right|} \int_{M_{j}} \boldsymbol{U}(x, y, t) d x d y$ is the approximation of the cell averages of the solution

at time $t$, and the quantity $\overline{\boldsymbol{S}}_{j}$ is a discretization of the cell averages of the source term, $\overline{\boldsymbol{S}}_{j} \approx \frac{1}{\left|M_{j}\right|} \int_{M_{j}} \boldsymbol{S}(\boldsymbol{U}(x, y, t), B(x, y)) d x d y$, which will be discussed in Section 4.

The semi-discrete scheme (4) uses the bottom elevation $B_{j k}:=B\left(P_{j k}\right)$ at the midpoint $P_{j k}$ of the $k$ th cell interface and the values $\boldsymbol{U}_{j}\left(P_{j k}\right)$ and $\boldsymbol{U}_{j k}\left(P_{j k}\right)$ at time $t$ on the two sides of this interface (inside and outside of the cell $M_{j}$, respectively), which are determined using the positivity preserving piecewise linear reconstructions in the cell $M_{j}$ and its neighboring cell $M_{j k}$, respectively; for details, see Sections 2.3, 2.4 and 3.

Finally, the one-sided local speeds of propagation at the $k$ th interface of the cell $M_{j}$ can be estimated using the smallest, $\lambda_{1}\left[V_{j k}\right]$, and largest, $\lambda_{3}\left[V_{j k}\right]$, eigenvalues of the Jacobian

$$
V_{j k}=\cos \left(\theta_{j k}\right) \frac{\partial \boldsymbol{F}}{\partial \boldsymbol{U}}+\sin \left(\theta_{j k}\right) \frac{\partial \boldsymbol{G}}{\partial \boldsymbol{U}}
$$


as follows:

$$
\begin{aligned}
& a_{j k}^{\text {in }}=-\min \left\{\lambda_{1}\left[V_{j k}\left(\boldsymbol{U}_{j}\left(P_{j k}\right)\right)\right], \lambda_{1}\left[V_{j k}\left(\boldsymbol{U}_{j k}\left(P_{j k}\right)\right)\right], 0\right\}, \\
& a_{j k}^{\text {out }}=\max \left\{\lambda_{3}\left[V_{j k}\left(\boldsymbol{U}_{j}\left(P_{j k}\right)\right)\right], \lambda_{3}\left[V_{j k}\left(\boldsymbol{U}_{j k}\left(P_{j k}\right)\right)\right], 0\right\} .
\end{aligned}
$$

Remark 2. If the value of $a_{j k}^{\text {in }}+a_{j k}^{\text {out }}$ in equation (4) is zero or very close to zero (smaller than $10^{-10}$ in all of our numerical experiments), we avoid division by zero or by a very small number using the following approximations:

$$
\begin{aligned}
\frac{\left[a_{j k}^{\text {in }} \boldsymbol{F}\left(\boldsymbol{U}_{j k}\left(P_{j k}\right), B_{j k}\right)+a_{j k}^{\text {out }} \boldsymbol{F}\left(\boldsymbol{U}_{j}\left(P_{j k}\right), B_{j k}\right)\right]}{a_{j k}^{\text {in }}+a_{j k}^{\text {out }}} & \approx \frac{\left[\boldsymbol{F}\left(\boldsymbol{U}_{j k}\left(P_{j k}\right), B_{j k}\right)+\boldsymbol{F}\left(\boldsymbol{U}_{j}\left(P_{j k}\right), B_{j k}\right)\right]}{2}, \\
\frac{\left[a_{j k}^{\text {in }} \boldsymbol{G}\left(\boldsymbol{U}_{j k}\left(P_{j k}\right), B_{j k}\right)+a_{j k}^{\text {out }} \boldsymbol{G}\left(\boldsymbol{U}_{j}\left(P_{j k}\right), B_{j k}\right)\right]}{a_{j k}^{\text {in }}+a_{j k}^{\text {out }}} & \approx \frac{\left[\boldsymbol{G}\left(\boldsymbol{U}_{j k}\left(P_{j k}\right), B_{j k}\right)+\boldsymbol{G}\left(\boldsymbol{U}_{j}\left(P_{j k}\right), B_{j k}\right)\right]}{2}, \\
\frac{a_{j k}^{\text {in }} a_{j k}^{\text {out }}}{a_{j k}^{\text {in }}+a_{j k}^{\text {out }}}\left[\boldsymbol{U}_{j k}\left(P_{j k}\right)-\boldsymbol{U}_{j}\left(P_{j k}\right)\right] & \approx 0 .
\end{aligned}
$$

These approximations are obtained by using similar extreme values of the directional local speeds of propagation of the waves at the cell interface inward and outward the computational cell. These values are used to delimit different domains in which the solution is still smooth and the domains in which the solution may not be smooth when it evolves from the time level $t^{n}$ to $t^{n+1}$. These domains are used in the procedure to obtain the semidiscrete form of the scheme as explained in details in [27] for triangular grids. The stability condition of the proposed central-upwind scheme using the semi-discrete form (4) and the approximations in Remark 2 will be discussed in Remark 8.

Remark 3. For very small values of the water depth $h$ we compute the velocity components at cell interfaces using the following desingularization formula proposed in [28]:

$$
(u, v)^{T}=\frac{\sqrt{2} h}{\sqrt{h^{4}+\max \left(h^{4}, \varepsilon\right)}}(u h, v h)^{T},
$$

where $\varepsilon$ is a small positive number which is taken in our numerical experiments equal to 
the square of the maximum of the computational cells areas in the entire domain. For consistency, we recompute the discharge values at cell interfaces using the obtained velocity values by $p:=h \cdot u$ and $q:=h \cdot v$, which are used in equations (4) to evaluate the fluxes.

Remark 4. The semi-discretization (4) is a system of ODEs, which has to be integrated in time using a sufficiently accurate and stable ODE solver. In all of the numerical experiments reported in Section 6, we have used the three-stage third-order strong stability preserving (SSP) Runge-Kutta method originally developed in [42]; see also [19, 20] for details on SSP ODE solvers. We note that one step of the three-stage third-order SSP Runge-Kutta method is a convex combination of three forward Euler steps: this property is useful to ensure the positivity preserving property of the resulting finite-volume method. We also note that even though the designed scheme is second-order accurate in space, we prefer to use the thirdorder-rather than the second-order-SSP method thanks to its larger stability domain and better nonlinear stability properties; see, e.g., [19, 20].

\subsection{Continuous Piecewise Linear Approximation of the Bottom}

Let us assume that the bottom topography function $B$ is a piecewise smooth function. In order to construct its continuous piecewise linear approximation, we first define the values of $B$ at the vertices $P_{j k_{i}}=\left(x_{j k_{i}}, y_{j k_{i}}\right)$ of the cell $M_{j}$. We use the following formula for the point value of the bottom topography at $P_{j k_{i}}$ :

$$
B_{j k_{i}}:=\frac{1}{2}\left[\lim _{\varepsilon \rightarrow 0} \max _{\|\zeta\|=\varepsilon} B\left(x_{j k_{i}}+\zeta_{x}, y_{j k_{i}}+\zeta_{y}\right)+\lim _{\varepsilon \rightarrow 0} \min _{\|\zeta\|=\varepsilon} B\left(x_{j k_{i}}+\zeta_{x}, y_{j k_{i}}+\zeta_{y}\right)\right]
$$

where $\zeta=\left(\zeta_{x}, \zeta_{y}\right)^{T}$. Note that this formula reduces to $B_{j k_{i}}:=B\left(x_{j k_{i}}, y_{j k_{i}}\right)$ if the function $B$ is continuous at $P_{j k_{i}}$.

We then obtain the approximate values of $B$ at the midpoint of the interface connecting 
the points $P_{j k_{1}}$ and $P_{j k_{2}}$ using a linear approximation resulting in

$$
B_{j k}=\frac{1}{2}\left(B_{j k_{1}}+B_{j k_{2}}\right)
$$

Equipped with the approximate values of $B$ at the midpoints of each cell interface, we approximate the value of $B$ at the center of mass $G_{j}$ by

$$
B_{j}=\frac{1}{\left|M_{j}\right|} \int_{M_{j}} B(x, y) d x d y \approx \sum_{k=1}^{m_{j}} \mu_{k} B_{j k}
$$

where $\mu_{k}=\mathcal{A}_{j k} /\left|M_{j}\right|$ and $\mathcal{A}_{j k}$ is the area of the triangle $G_{j} P_{j k_{1}} P_{j k_{2}}$ (see Figure 1). Given the values at the vertices, $B_{j}, B_{j k_{1}}$ and $B_{j k_{2}}$, we obtain a linear approximation of $B$ over this triangle.

Finally, we obtain the continuous piecewise linear approximation of $B$ at the cell $M_{j}$ by taking the union of $m_{j}$ planes over the corresponding triangles connecting the two neighboring vertices of $M_{j}$ and its center of mass.

\subsection{Piecewise Linear Reconstruction}

In order to obtain a piecewise linear reconstruction of $w, p$ and $q$, we need to approximate its gradients in each cell. The gradient of the $i$ th component $\boldsymbol{U}^{(i)}$ of the vector $\boldsymbol{U}$ in the cell $M_{j}$, denoted by $\nabla \boldsymbol{U}_{j}^{(i)}(i=1,2,3)$, is computed by averaging it over $M_{j}$ using the Green-Gauss theorem as follows:

$$
\nabla \boldsymbol{U}_{j}^{(i)}=\frac{1}{\left|M_{j}\right|} \int_{M_{j}} \nabla \boldsymbol{U}_{j}^{(i)}(x, y) d x d y=\frac{1}{\left|M_{j}\right|} \sum_{s=1}^{m_{j}} \int_{\left(\partial M_{j}\right)_{s}} \tilde{\boldsymbol{U}}_{j s}^{(i)} n_{j s} d \Gamma
$$

where $\widetilde{\boldsymbol{U}}_{j s}^{(i)}$ is the estimated value of $\boldsymbol{U}^{(i)}$ at the cell interface $\left(\partial M_{j}\right)_{s}$ and $d \Gamma$ denotes an infinitesimal boundary arclength.

To prevent oscillations, we propose a new minmod-type reconstruction which is more 
suitable for cell-vertex finite-volume schemes. To this end, we compute $m_{j}$ gradients. The $k$ th gradient $\left(k \in\left\{1, \ldots, m_{j}\right\}\right)$ is calculated using equation $(7)$, where the estimated values $\widetilde{\boldsymbol{U}}_{j s}^{(i)}$ on the cell interfaces $\left(\partial M_{j}\right)_{s}$ are obtained using the following three-step procedure:

- First, we take $\widetilde{\boldsymbol{U}}_{j s}^{(i)}=\left(\overline{\boldsymbol{U}}_{j}^{(i)}+\overline{\boldsymbol{U}}_{j s}^{(i)}\right) / 2$ for $i=1,2,3$ and for all $s$ except for $s=k$,

- In the case $s=k$, we use

$$
\widetilde{\boldsymbol{U}}_{j, k}^{(i)}=\left(\widetilde{\boldsymbol{U}}_{j, k+1}^{(i)}+\widetilde{\boldsymbol{U}}_{j, k-1}^{(i)}\right) / 2, \quad i=1,2,3,
$$

where we set $\widetilde{\boldsymbol{U}}_{j, 0}^{(i)}:=\widetilde{\boldsymbol{U}}_{j, m_{j}}^{(i)}$ and $\widetilde{\boldsymbol{U}}_{j, m_{j}+1}^{(i)}:=\widetilde{\boldsymbol{U}}_{j, 1}^{(i)}$,

- Then, the obtained values of $\widetilde{\boldsymbol{U}}_{j s}^{(1)}=\widetilde{w}_{j s}$ are corrected: If $\widetilde{w}_{j s}<B_{j s}$, that is, if the estimated value of $w$ is below the bottom elevation at the midpoint of the sth cell interface, we raise that value to $\widetilde{w}_{j s}:=B_{j s}$.

Finally, for each variable $w, h u$ and $h v$, out of the $m_{j}$ gradient values we select the one that has the smallest magnitude and use the obtained numerical gradients $\nabla \boldsymbol{U}_{j}=\left(\left(\boldsymbol{U}_{x}\right)_{j},\left(\boldsymbol{U}_{y}\right)_{j}\right)^{T}$ to build the corresponding linear pieces in the cell $M_{j}$ :

$$
\boldsymbol{U}_{j}(x, y):=\overline{\boldsymbol{U}}_{j}+\left(\boldsymbol{U}_{x}\right)_{j}\left(x-x_{j}\right)+\left(\boldsymbol{U}_{y}\right)_{j}\left(y-y_{j}\right)
$$

The values $\boldsymbol{U}_{j}\left(P_{j k}\right)$ required in (4), (5) are then obtained by substituting the coordinates of $P_{j k}$ into (8).

Remark 5. Note that the reconstruction (8) satisfies the relationship similar to (6), established for the continuous piecewise linear reconstruction of the bottom topography in Section 2.3. In particular, for the water surface elevation $w$ and the water depth $h:=w-B$ we have

$$
\sum_{k=1}^{m_{j}} \mu_{k} w\left(P_{j k}\right)=\bar{w}_{j}, \quad \sum_{k=1}^{m_{j}} \mu_{k} h\left(P_{j k}\right)=\bar{h}_{j},
$$


which will be used in the proof of positivity preserving property of the scheme presented in Section 5 .

We would like to point out that the piecewise linear reconstruction procedure for $w$ presented in this section does not guarantee positivity of the reconstructed values of $h$. Therefore, this reconstruction has to be corrected to preserve the positivity of $h$.

\section{Positivity Preserving Reconstruction for Water Surface Elevation}

In this section, we propose an algorithm for the positivity preserving reconstruction of $w$. We say that the reconstruction is positivity preserving if it leads to nonnegative computed values of water depth at all of the cell vertices. The obtained reconstruction can be viewed as a correction of the basic piecewise linear reconstruction

$$
w_{j}(x, y):=\bar{w}_{j}+\left(w_{x}\right)_{j}\left(x-x_{j}\right)+\left(w_{y}\right)_{j}\left(y-y_{j}\right)=\bar{w}_{j}+\nabla w_{j} \cdot\left(x-x_{j}, y-y_{j}\right)^{T},
$$

where the gradient $\nabla w_{j}=\left(\left(w_{x}\right)_{j},\left(w_{y}\right)_{j}\right)^{T}$ is calculated using the modified minmod-type limiter described in Section 2.4.

We will distinguish between the three cases depending on the amount of water present in the cell $M_{j}$ and on the local properties of the piecewise linear bottom approximation.

Case 1 (Wet Cells). We first consider the cells in which the water surface elevation $\bar{w}_{j}$ is greater than or equal to the bed elevation at all of the vertices of the cell $M_{j}$, that is, $\bar{w}_{j} \geq B_{j k_{1}}$ for all $k \in\left[1, m_{j}\right]$. In this case, it is possible to construct a single-plane reconstruction over the entire cell $M_{j}$. The reconstruction will take the form

$$
w_{j}(x, y):=\bar{w}_{j}+\alpha \nabla w_{j} \cdot\left(x-x_{j}, y-y_{j}\right)^{T}
$$


where a proper selection of the parameter $\alpha \in[0,1]$ will help to respect positivity of the water depth.

To obtain the values of water surface elevation and water depth at the cell vertices (denoted by $P_{j k_{1}}$ ) we use

$$
w_{j k_{1}}=\bar{w}_{j}+\alpha \nabla w_{j} \cdot \overrightarrow{G_{j} P_{j k_{1}}}
$$

and

$$
h_{j k_{1}}=w_{j k_{1}}-B_{j k_{1}}=\bar{w}_{j}-B_{j k_{1}}+\alpha \nabla w_{j} \cdot \overrightarrow{G_{j} P_{j k_{1}}}
$$

The condition that the water surface elevation is greater than or equal to the bed elevation at all of the vertices of the cell $M_{j}$ implies that the set of parameters $\alpha \in[0,1]$ which guarantee the positivity of $h_{j k_{1}}$ at all of the cell vertices, is not empty since it contains $\alpha=0$. We then consider the largest $\alpha$ in this set denoted by $\alpha_{\max }$ and we use the singleplane reconstruction based on the gradient $\alpha_{\max } \nabla w_{j}$. The parameter $\alpha_{\max }$ can be easily obtained by requiring $h_{j k_{1}} \geq 0$ in (12) for all $k_{1}$.

Case 2 (Partially Wet Cells with the Possibility of Single-Plane Reconstruction (10)). The second possible case corresponds to the situation, in which there are some cell vertices $P_{j k_{1}}$ for which $\bar{w}_{j}<B_{j k_{1}}$. We split the vertices $P_{j k_{1}}, k=1, \ldots, m_{j}$ into two separate sets: wet vertices where $\bar{w}_{j} \geq B_{j k_{1}}$, and dry vertices where $\bar{w}_{j}<B_{j k_{1}}$. Due to (9) and since $\bar{w}_{j} \geq B_{j}$, the set of wet vertices is not empty.

Similar to Case 1, we consider the parameter $\alpha_{\max }$ such that for all $\alpha \in\left[0, \alpha_{\max }\right]$ the values of the water depth obtained using equation (12) are nonnegative for all wet vertices. If $h$ is also nonnegative for $\alpha=\alpha_{\max }$ at all of the dry vertices, we use $\alpha=\alpha_{\max }$ for the single-plane reconstruction (11), (12). Otherwise, no single-plane positivity preserving reconstruction is possible and we build a reconstruction consisting of $m_{j}$ planes defined over the cell $M_{j}$. 
Case 3 (Partially Wet Cells Not Included in Case 2). In this case, we propose a reconstruction with the minimal deviation from the direction of the initial gradient $\nabla w_{j}$.

Case 3a: We first consider partially wet cells with only one dry vertex $P_{j k_{1}}$ (that is, $\left.\bar{w}_{j}<B_{j k_{1}}\right)$. In the reconstruction, we set zero water depth at this point (that is, we set $\left.w\left(P_{j k_{1}}\right):=B_{j k_{1}}\right)$ and since the linear reconstruction should also satisfy $w\left(G_{j}\right)=\bar{w}_{j}$, we only need a third point to complete the reconstruction. To this end, we consider $m_{j}-1$ planes passing through these two points and the point $\left(P_{j k_{1}^{\prime}}, B_{j k_{1}^{\prime}}\right)$ for $k_{1}^{\prime} \neq k_{1}$ and we compute their gradients $\left\{\nabla w_{k_{1}^{\prime}}\right\}$. We then consider only those gradients that lead to positive reconstructions and out of them select the gradient which has the minimal deviation from the direction of the initial gradient $\nabla w_{j}$ by computing the angles between $\nabla w_{j}$ and $\nabla w_{k_{1}^{\prime}}$. If none of the gradients $\nabla w_{k_{1}^{\prime}}$ guarantees a positive reconstruction, we proceed with Case $3 \mathrm{~b}$.

Case 3b: Finally, we consider partially wet cells not covered by Case 3a. We now use a union of $m_{j}$ planes defined over the cell $M_{j}$. First, we set zero depth at the cell vertices at which the condition $\bar{w}_{j}<B_{j k_{1}}$ is satisfied. There are many possibilities for the reconstruction, but in order to avoid oscillations we consider the constant depth denoted by $\widehat{h}_{j}$ at the other vertices where $\bar{w}_{j} \geq B_{j k_{1}}$. The value of $\widehat{h}_{j}$ can be obtained using the conservation requirement (9) as follows:

$$
\widehat{h}_{j}=\frac{\bar{h}_{j}}{\sum_{k=1}^{m_{j}} \mu_{k} \varepsilon_{k}}, \quad \text { where } \quad \varepsilon_{k}= \begin{cases}1, & \text { if } \bar{w}_{j} \geq B_{j k_{1}} \text { and } \bar{w}_{j} \geq B_{j k_{2}}, \\ 0, & \text { if } \bar{w}_{j}<B_{j k_{1}} \text { and } \bar{w}_{j}<B_{j k_{2}}, \\ 1 / 2, & \text { otherwise. }\end{cases}
$$

Remark 6. In all of the cases considered above, the values of water surface elevation and 
water depth at the midpoints $P_{j k}$ are obtained from the values at the cell vertices by

$$
w\left(P_{j k}\right)=\frac{w\left(P_{j k_{1}}\right)+w\left(P_{j k_{2}}\right)}{2}, \quad h\left(P_{j k}\right)=\frac{h\left(P_{j k_{1}}\right)+h\left(P_{j k_{2}}\right)}{2} .
$$

Therefore, if the reconstructed water depth is nonnegative at all of the cell vertices, it will be also nonnegative over the entire cell, and in particular, at the midpoints of its interfaces. The positivity of the water depth at the midpoints of the interfaces will be crucial in the proof of the positivity preserving property of the scheme presented in Section 5.

\section{Well-Balanced Discretization of the Source Term}

The proposed semi-discrete central-upwind scheme (4) includes the cell average of the source term $\overline{\boldsymbol{S}}_{j} \equiv\left(0, \bar{S}_{j}^{(2)}, \bar{S}_{j}^{(3)}\right)^{T}$. To design a well-balanced scheme, that is, a scheme that exactly preserves "lake at rest" steady-state solutions satisfying $w \equiv C, u \equiv v \equiv 0$, where $C$ is a constant, a special quadrature has to be designed.

Note that for a given "lake at rest" solution, $\boldsymbol{U}_{j}\left(P_{j k}\right)=\boldsymbol{U}_{j k}\left(P_{j k}\right)=(C, 0,0)^{T}$, and the two momentum equations of the semi-discrete scheme (4) reduce to

$$
\begin{aligned}
& -\frac{g}{2\left|M_{j}\right|} \sum_{k=1}^{m_{j}} \ell_{j k} \cos \left(\theta_{j k}\right)\left(C-B_{j k}\right)^{2}+\bar{S}_{j}^{(2)}=0, \\
& -\frac{g}{2\left|M_{j}\right|} \sum_{k=1}^{m_{j}} \ell_{j k} \sin \left(\theta_{j k}\right)\left(C-B_{j k}\right)^{2}+\bar{S}_{j}^{(3)}=0 .
\end{aligned}
$$

In the remaining part of the section, we derive a quadrature that satisfies the well-balancing conditions (13).

First, the source term $\bar{S}_{j}^{(2)}$ can be rewritten in the following form using the divergence 
theorem:

$$
\begin{aligned}
\bar{S}_{j}^{(2)} & =-\frac{g}{\left|M_{j}\right|} \int_{M_{j}}(w-B) B_{x} d x d y=\frac{g}{2\left|M_{j}\right|} \int_{M_{j}}\left((w-B)^{2}\right)_{x} d x d y-\frac{g}{\left|M_{j}\right|} \int_{M_{j}}(w-B) w_{x} d x d y \\
& =\frac{g}{2\left|M_{j}\right|} \sum_{k=1}^{m_{j}} \int_{\left(\partial M_{j}\right)_{k}}(w-B)^{2} \cos \left(\theta_{j k}\right) d s-\frac{g}{\left|M_{j}\right|} \int_{M_{j}}(w-B) w_{x} d x d y
\end{aligned}
$$

We then apply the midpoint rule to approximate the integrals on the right-hand side (RHS) of (14) to obtain the well-balanced quadrature for $\bar{S}_{j}^{(2)}$ :

$$
\bar{S}_{j}^{(2)}=\frac{g}{2\left|M_{j}\right|} \sum_{k=1}^{m_{j}} \ell_{j k}\left(w_{j}\left(P_{j k}\right)-B_{j k}\right)^{2} \cos \left(\theta_{j k}\right)-g\left(w_{x}\right)_{j}\left(\bar{w}_{j}-B_{j}\right) .
$$

Similarly, the well-balanced quadrature for the source term $\bar{S}_{j}^{(3)}$ is

$$
\bar{S}_{j}^{(3)}=\frac{g}{2\left|M_{j}\right|} \sum_{k=1}^{m_{j}} \ell_{j k}\left(w_{j}\left(P_{j k}\right)-B_{j k}\right)^{2} \sin \left(\theta_{j k}\right)-g\left(w_{y}\right)_{j}\left(\bar{w}_{j}-B_{j}\right) .
$$

Indeed, the quadratures (15) and (16) are well-balanced since the terms on the RHS of (15) and (16) containing the derivatives $\left(w_{x}\right)_{j}$ and $\left(w_{y}\right)_{j}$ vanish for the "lake at rest" solution $\boldsymbol{U} \equiv(C, 0,0)^{T}$, and the well-balancing conditions (13) are satisfied.

\section{Positivity Preserving Property of the Scheme}

In this section, we prove the positivity preserving property of the proposed centralupwind scheme.

Theorem 1. Consider the semi-discrete central-upwind scheme (4) for the Saint-Venant system (2), (3). Let the ODE system (4) is integrated using the forward Euler method. We assume that at time $t=t^{n}$ the computed water depth is nonnegative, that is, $\bar{w}_{j}^{n} \geq B_{j}$ for 
all $j$ and that the time step size is restricted by

$$
\Delta t \leq \frac{1}{2 a} \min _{j, k}\left\{d_{j k}\right\}
$$

where $a=\max \left\{a_{j k}^{\text {in }}, a_{j k}^{\text {out }}\right\}$ and $d_{j k}$ is the distance between the center of mass $G_{j}$ of the cell $M_{j}$ and its $k$ th interface $P_{j k_{1}} P_{j k_{2}}$.

Then $\bar{w}_{j}^{n+1} \geq B_{j}$ for all $j$ at time $t=t^{n+1}$.

Proof: Applying the forward Euler temporal discretization to the first equation in (4) yields

$$
\begin{aligned}
\bar{w}_{j}^{n+1}=\bar{w}_{j}^{n} & -\frac{\Delta t}{\left|M_{j}\right|} \sum_{k=1}^{m_{j}} \frac{\ell_{j k} \cos \left(\theta_{j k}\right)}{a_{j k}^{\text {in }}+a_{j k}^{\text {out }}}\left[a_{j k}^{\text {in }}(h u)_{j k}\left(P_{j k}\right)+a_{j k}^{\text {out }}(h u)_{j}\left(P_{j k}\right)\right] \\
& -\frac{\Delta t}{\left|M_{j}\right|} \sum_{k=1}^{m_{j}} \frac{\ell_{j k} \sin \left(\theta_{j k}\right)}{a_{j k}^{\text {in }}+a_{j k}^{\text {out }}}\left[a_{j k}^{\text {in }}(h v)_{j k}\left(P_{j k}\right)+a_{j k}^{\text {out }}(h v)_{j}\left(P_{j k}\right)\right] \\
& +\frac{\Delta t}{\left|M_{j}\right|} \sum_{k=1}^{m_{j}} \ell_{j k} \frac{a_{j k}^{\text {in }} a_{j k}^{\text {out }}}{a_{j k}^{\text {in }}+a_{j k}^{\text {out }}}\left[w_{j k}\left(P_{j k}\right)-w_{j}\left(P_{j k}\right)\right] .
\end{aligned}
$$

Recall that the reconstruction proposed in Sections 2.4 and 3 guarantees that the water surface elevation and the water depth satisfy the following equalities at time level $t=t^{n}$ :

$$
\bar{w}_{j}^{n}=\sum_{k=1}^{m_{j}} \mu_{k} w_{j}\left(P_{j k}\right), \quad \bar{h}_{j}^{n}=\sum_{k=1}^{m_{j}} \mu_{k} h_{j}\left(P_{j k}\right)
$$

and the inequalities $h_{j}\left(P_{j k}\right) \geq 0$ and $h_{j k}\left(P_{j k}\right) \geq 0$.

Since the piecewise linear reconstruction of the bottom topography is continuous we have $w_{j k}\left(P_{j k}\right)-w_{j}\left(P_{j k}\right)=h_{j k}\left(P_{j k}\right)-h_{j}\left(P_{j k}\right)$ for each $k \in\left[0, m_{j}\right]$. We then use this equality 
together with (19) to rewrite equation (18) in the following form:

$$
\begin{aligned}
\bar{h}_{j}^{n+1} & =\frac{\Delta t}{\left|M_{j}\right|} \sum_{k=1}^{m_{j}} h_{j k}\left(P_{j k}\right) \frac{\ell_{j k} a_{j k}^{\text {in }}}{a_{j k}^{\text {in }}+a_{j k}^{\text {out }}}\left[a_{j k}^{\text {out }}-u_{j k}^{\theta}\left(P_{j k}\right)\right] \\
& +\sum_{k=1}^{m_{j}} h_{j}\left(P_{j k}\right)\left(\mu_{k}-\frac{\Delta t}{\left|M_{j}\right|} \cdot \frac{\ell_{j k} a_{j k}^{\text {out }}}{a_{j k}^{\text {in }}+a_{j k}^{\text {out }}}\left[a_{j k}^{\text {in }}+u_{j}^{\theta}\left(P_{j k}\right)\right]\right),
\end{aligned}
$$

where

$u_{j k}^{\theta}\left(P_{j k}\right):=\cos \left(\theta_{j k}\right) u_{j k}\left(P_{j k}\right)+\sin \left(\theta_{j k}\right) v_{j k}\left(P_{j k}\right), \quad u_{j}^{\theta}\left(P_{j k}\right):=\cos \left(\theta_{j k}\right) u_{j}\left(P_{j k}\right)+\sin \left(\theta_{j k}\right) v_{j}\left(P_{j k}\right)$.

Since $a_{j k}^{\text {out }} \geq u_{j k}^{\theta}\left(P_{j k}\right)$ and $h_{j k}\left(P_{j k}\right) \geq 0$, the first term on the RHS of (20) is nonnegative. Since

$$
\frac{\Delta t}{\left|M_{j}\right|} \cdot \frac{\ell_{j k} a_{j k}^{\text {out }}}{a_{j k}^{\text {in }}+a_{j k}^{\text {out }}}\left[a_{j k}^{\text {in }}+u_{j}^{\theta}\left(P_{j k}\right)\right] \leq \frac{\Delta t}{\left|M_{j}\right|} \ell_{j k} a_{j k}^{\text {out }} \text { and } \quad h_{j}\left(P_{j k}\right) \geq 0
$$

the second term on the RHS of (20) is positive provided that

$$
\Delta t \leq \frac{\mu_{k}\left|M_{j}\right|}{\ell_{j k} a_{j k}^{\text {out }}}
$$

This condition is valid and can be verified using the time step restriction (17), $\mu_{k}=\mathcal{A}_{j k} /\left|M_{j}\right|$ and $\mathcal{A}_{j k}=d_{j k} \ell_{j k} / 2$.

Remark 7. The proof of Theorem 1 is still valid if the forward Euler temporal discretization is replaced with a high-order SSP ODE solver, since one step of any SSP method consists of a convex combination of several forward Euler steps.

Remark 8. We note that the condition (17) only ensures the positivity preserving property of the designed scheme, but does not a-priori guarantees its stability. Similar to the stability requirement of the central-upwind scheme on the triangular meshes $([6,27])$, we can formulate the CFL condition for the proposed cell-vertex central-upwind scheme: No nonlinear (possibly discontinuous) waves generated at the cell interfaces should reach the 
center of mass of the computational cell over a time step $\Delta t$. This leads to the following time step restriction:

$$
\Delta t<\frac{1}{a} \min _{j, k}\left\{d_{j k}\right\}
$$

which is less restrictive than (17). Therefore, the condition (17) is expected to ensure both the stability and positivity. Note that if the approximation given in Remark 2 for the proposed semi-discrete scheme is used, the condition (21) required for positivity is satisfied since in this case the value of the directional local speed $a_{j k}^{\text {out }}$ is very small.

\section{Numerical Examples}

In this section, we demonstrate the performance of the proposed central-upwind scheme on a variety of benchmarks. In all of the numerical experiments, we take $g=1$ except for Example 4, where we set $g=9.812$. In Examples 1-3, the proposed scheme is employed to compute small perturbations of the "lake at rest" steady states in different contexts. In Example 4, we simulate a rapidly varying flow arising in modeling dam breaking over discontinuous bottom topography. In Example 5, the exact solution for parabolic waves proposed in [43] is used for assessing the proposed method. We have used this analytical solution to test the accuracy and positivity of the proposed scheme.

\section{Example 1-Small Perturbation over an Exponential Hump}

In the first example, we consider the benchmark originally proposed in [31] and then widely used in the literature (see, e.g., [7, 23]), as well as its more challenging version ([6]), in which a very small perturbation of the water surface elevation is considered. We study the ability of the cell-vertex central-upwind scheme to accurately capture the propagation of a small perturbation of the "lake at rest" steady state over an exponential hump described by

$$
B(x, y)=0.8 \exp \left(-5(x-0.9)^{2}-50 y^{2}\right)
$$


The computational domain is $[0,2] \times[-0.5,0.5]$, and the water surface is initially at rest everywhere except for the stripe $0.05<x<0.15$, where a small perturbation is initially located:

$$
w(x, y, 0)=\left\{\begin{array}{ll}
1+\varepsilon, & 0.05<x<0.15, \\
1, & \text { otherwise }
\end{array} \quad u(x, y, 0) \equiv v(x, y, 0) \equiv 0 .\right.
$$

First, we take a relatively large perturbation $\varepsilon=0.01$ and compute the solution using the grid with an average cell area $\left|M_{j}\right|=2.24 \cdot 10^{-5}$. The evolution (at times $t=0.6,0.9$, $1.2,1.5$ and 1.8) of the right-going portion of the water surface perturbation is shown in the left column of Figure 2. As one can see, the obtained solution is oscillation-free and the achieved resolution is comparable to the resolution achieved in $[6,23,31]$. To further verify the robustness of the proposed method, we take smaller perturbation values $\varepsilon=10^{-3}$ and $\varepsilon=10^{-4}$ and compute the solution at the same time moments $t=0.6,0.9,1.2,1.5$ and 1.8 using the grids with average cell areas $\left|M_{j}\right|=1.25 \cdot 10^{-5}$ and $\left|M_{j}\right|=7.78 \cdot 10^{-6}$, respectively. The obtained results are shown in the right column of Figure 2 and in Figure 3. It should be observed that the computed solutions are still oscillation-free and highly resolved. This demonstrates the ability of the scheme to accurately capture quasi-steady states.

Next, we demonstrate the importance of the proposed well-balanced discretization of the source term. To this end, we design a non well-balanced cell-vertex central-upwind scheme by replacing the well-balanced quadratures (15) and (16) with the midpoint rule:

$$
\bar{S}_{j}^{(2)}=-g\left(\bar{w}_{j}-B_{j}\right)\left(B_{x}\right)_{j}, \quad \bar{S}_{j}^{(3)}=-g\left(\bar{w}_{j}-B_{j}\right)\left(B_{y}\right)_{j}
$$

where the components of $\nabla B$ are obtained using the divergence theorem:

$$
\left(B_{x}\right)_{j}=\frac{1}{\left|M_{j}\right|} \sum_{k=1}^{m_{j}} \ell_{j k} B_{j k} \cos \left(\theta_{j k}\right), \quad\left(B_{y}\right)_{j}=\frac{1}{\left|M_{j}\right|} \sum_{k=1}^{m_{j}} \ell_{j k} B_{j k} \sin \left(\theta_{j k}\right) .
$$



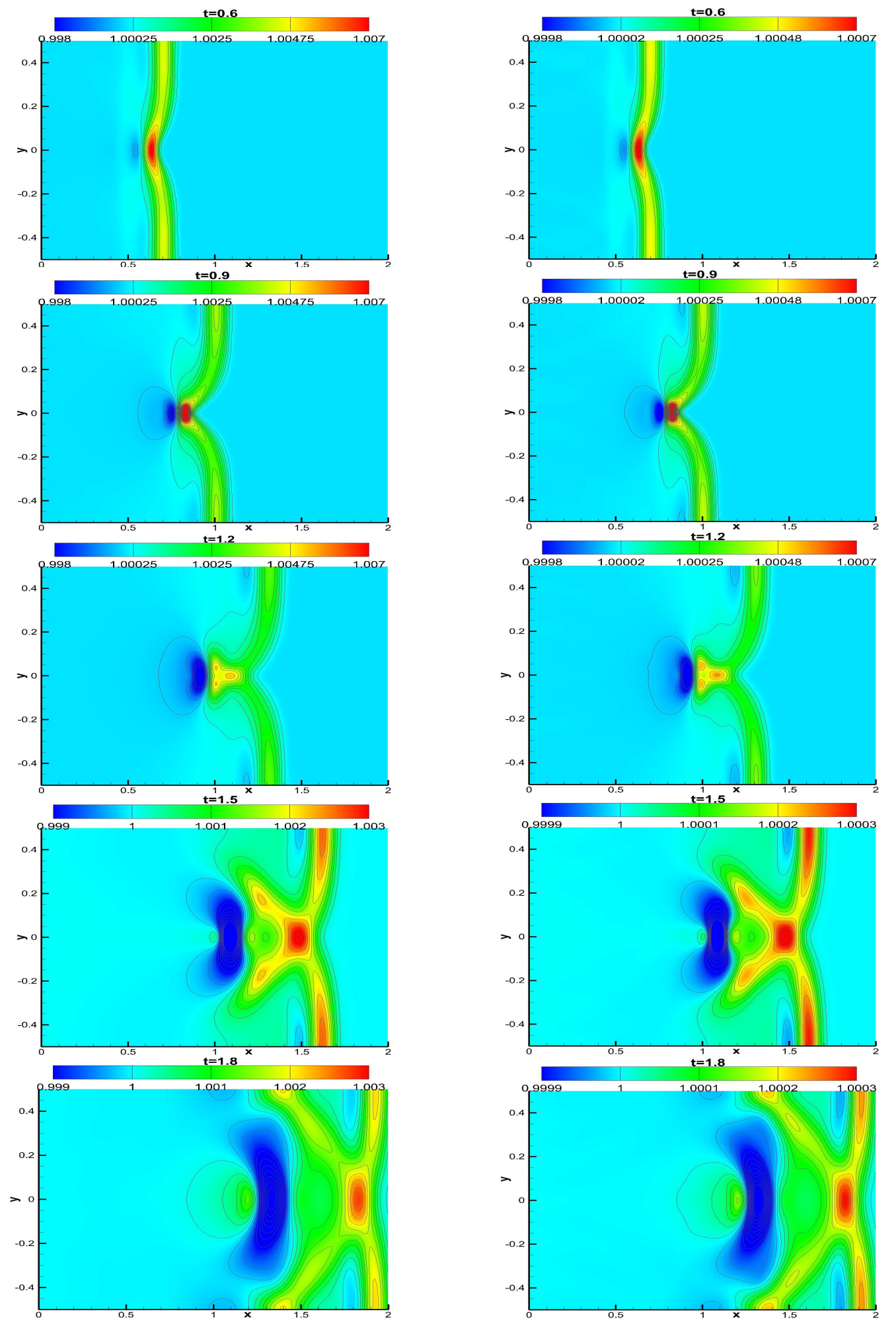

Figure 2: Example 1: Solution $(w)$ computed by the well-balanced cell-vertex central-upwind scheme for $\varepsilon=10^{-2}$ (left) and $\varepsilon=10^{-3}$ (right). 

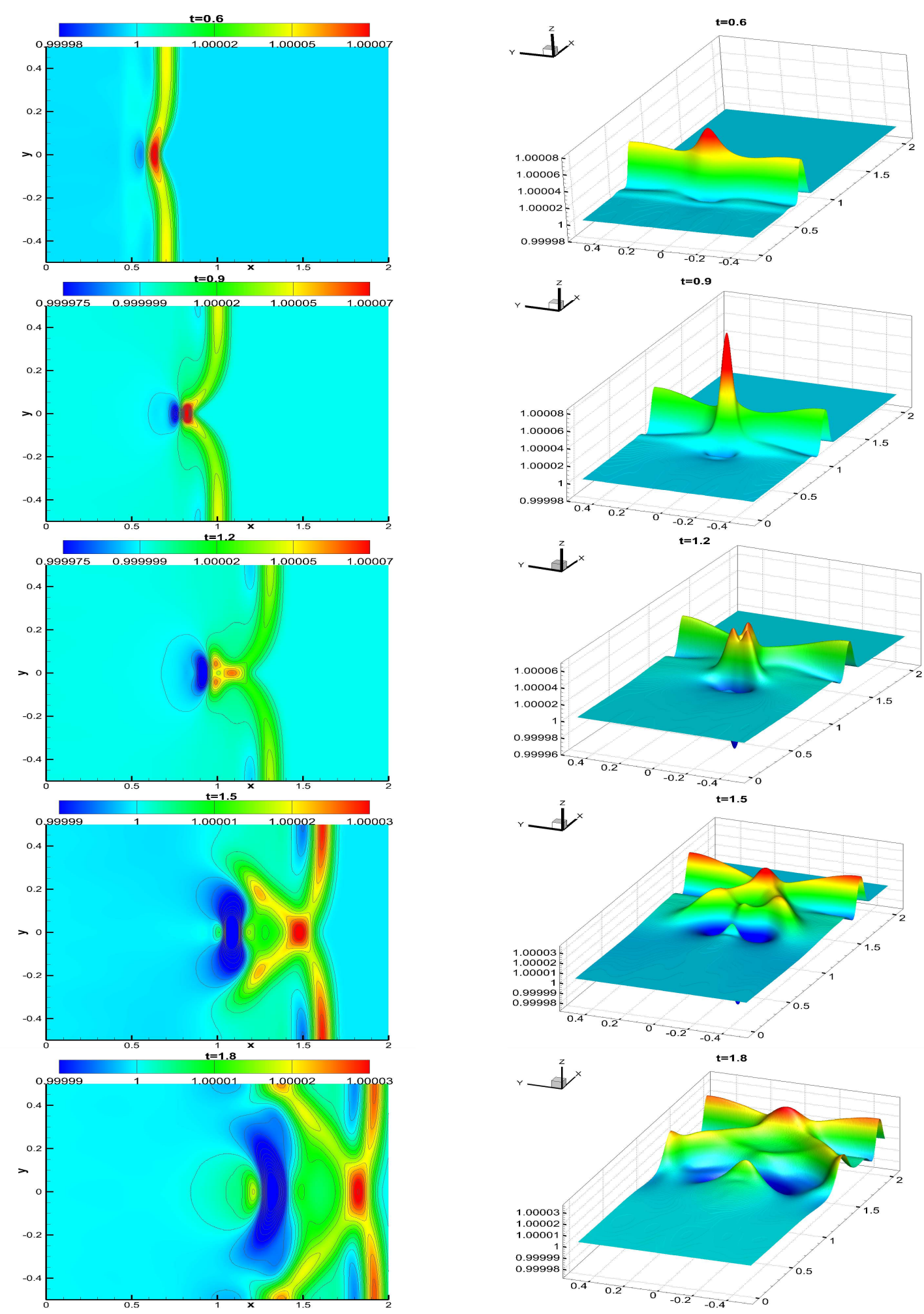

Figure 3: Example 1: Top (left) and three-dimensional (3-D) (right) views of the solution $(w)$ computed by the well-balanced cell-vertex central-upwind scheme for $\varepsilon=10^{-4}$. 
The non well-balanced solution computed for $\varepsilon=10^{-4}$ at time $t=0.6$ on the same grid as before (with average cell areas $\left|M_{j}\right|=7.78 \cdot 10^{-6}$ ) is shown in Figure 4. As one can see, the use of the non well-balanced scheme leads to spurious modes appearing at the plateau area which deform the solution. When we take a coarser mesh with average cell areas $\left|M_{j}\right|=2.24 \cdot 10^{-5}$, the non well-balanced solution is severely deformed and is completely incorrect, see Figure 4 (middle). The well-balanced scheme applied on the same coarse mesh leads, on the contrary, to oscillation-free results as it is shown in Figure 4 (right). This clearly demonstrates a crucial role of a well-balanced source term quadrature.
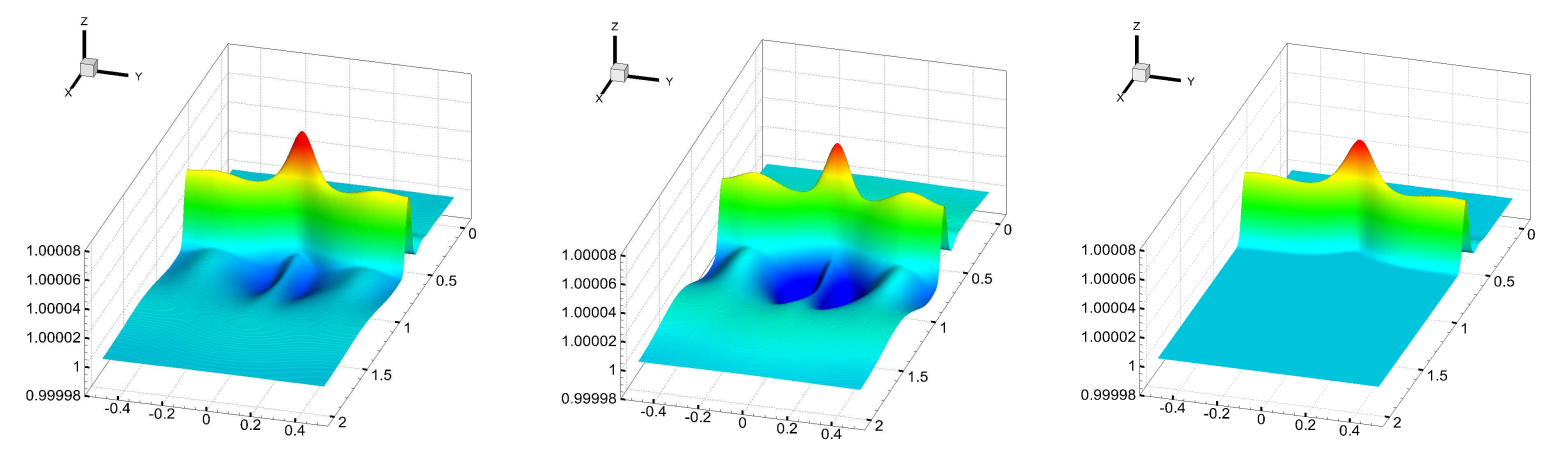

Figure 4: Example 1: Solutions $(w)$ computed by the non well-balanced cell-vertex central-upwind scheme using the fine (left) and coarse (middle) grids and by the well-balanced cell-vertex central-upwind scheme using the coarse grid (right). Here, $\varepsilon=10^{-4}$.

\section{Example 2: Small Perturbation over Submerged Flat Plateau}

In this example, we consider a submerged flat plateau as shown in Figure 5 (left). To further verify well-balanced and positivity preserving features of the proposed cell-vertex central-upwind scheme, we consider a slight modification of the test problem from [6], in which a small perturbation of the "lake at rest" steady state propagates over a submerged flat plateau located very close to the water surface. 
The computational domain is $[0,1] \times[-0.5,0.5]$ and the bottom topography is given by

$$
B(x, y)= \begin{cases}1-2 \varepsilon, & r \leq 0.1, \\ 10(1-2 \varepsilon)(0.2-r), & 0.1 \leq r \leq 0.2, \quad r:=\sqrt{(x-0.5)^{2}+y^{2}} \\ 0, & \text { otherwise }\end{cases}
$$

The outflow boundary conditions are used in the $x$-direction, while the wall boundary conditions are imposed in the $y$-direction. As in Example 1, the initial conditions correspond to a small perturbation of the "lake at rest" steady state:

$$
w(x, y, 0)=\left\{\begin{array}{ll}
1+\varepsilon, & 0.1<x<0.2, \\
1, & \text { otherwise }
\end{array} \quad u(x, y, 0) \equiv v(x, y, 0) \equiv 0 .\right.
$$

The value of the desingularization parameter $\varepsilon=0.01$ is used here.

The solution is computed using the proposed cell-vertex central-upwind scheme with average cell areas $\left|M_{j}\right|=2.24 \times 10^{-5}$. Figure 6 shows $w$ computed at times $t=0.2,0.35$ and 0.65. As one can see, no oscillations are observed and the positivity of the water depth is preserved. We then compute the solution on the same grid at the same times, but using the non well-balanced central-upwind scheme described in Example 1. The obtained results are presented in Figure 7, where spurious deformations are clearly observed. These deformations lead to numerical oscillations and widely increase if a coarser mesh is used.

\section{Example 3: Small Perturbation Bending around a Round-Shape Island}

This example, which is also a slight modification of the problem from [6], is designed to examine both well-balanced and positivity preserving properties of the studied scheme by testing its ability to handle a situation with a small perturbation of a "lake at rest" state propagating around an island. The round-shape island (see Figure 5, right) is represented 

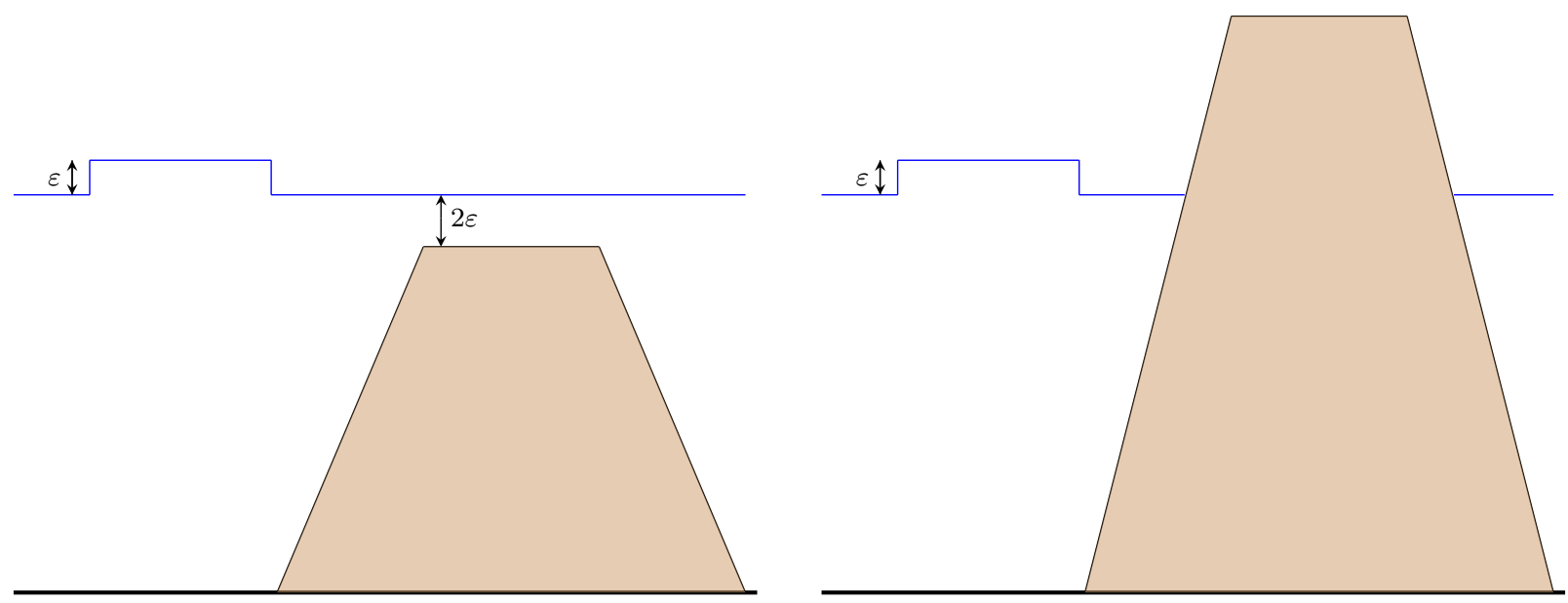

Figure 5: Examples 2 and 3: One-dimensional slices of the bottom topographies (24), left, and (25), right. These plots are not to scale.
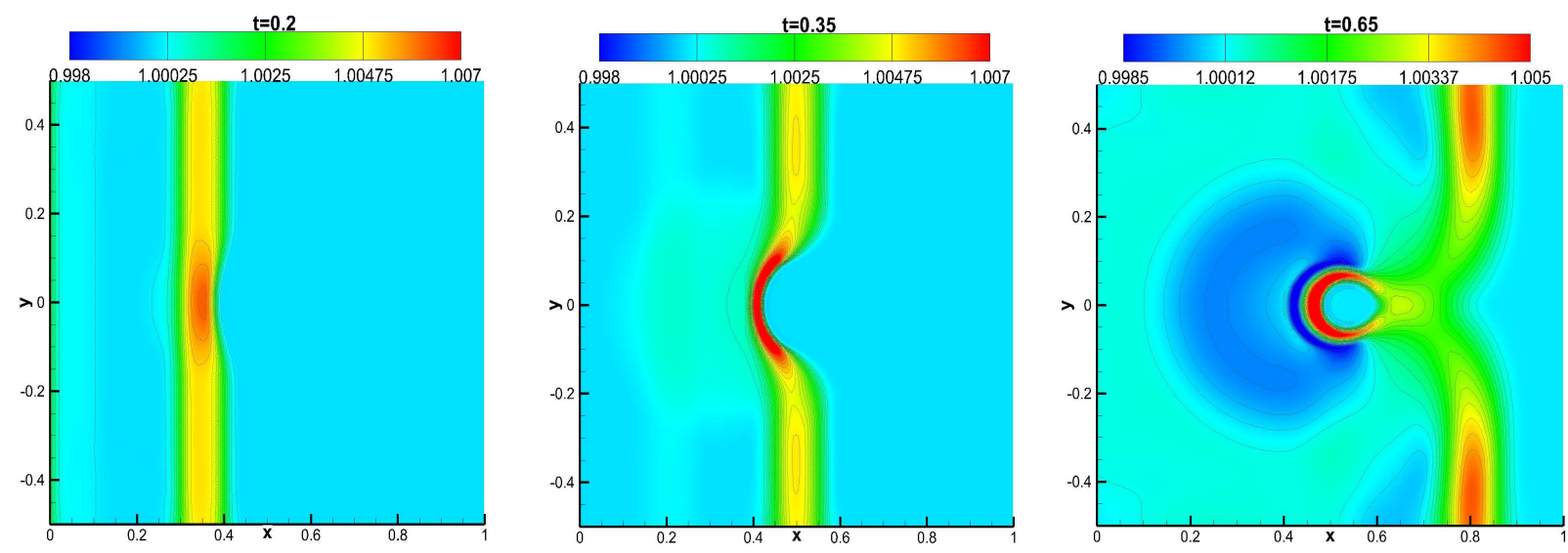

Figure 6: Example 2: Solution $(w)$ computed by the well-balanced cell-vertex central-upwind scheme.
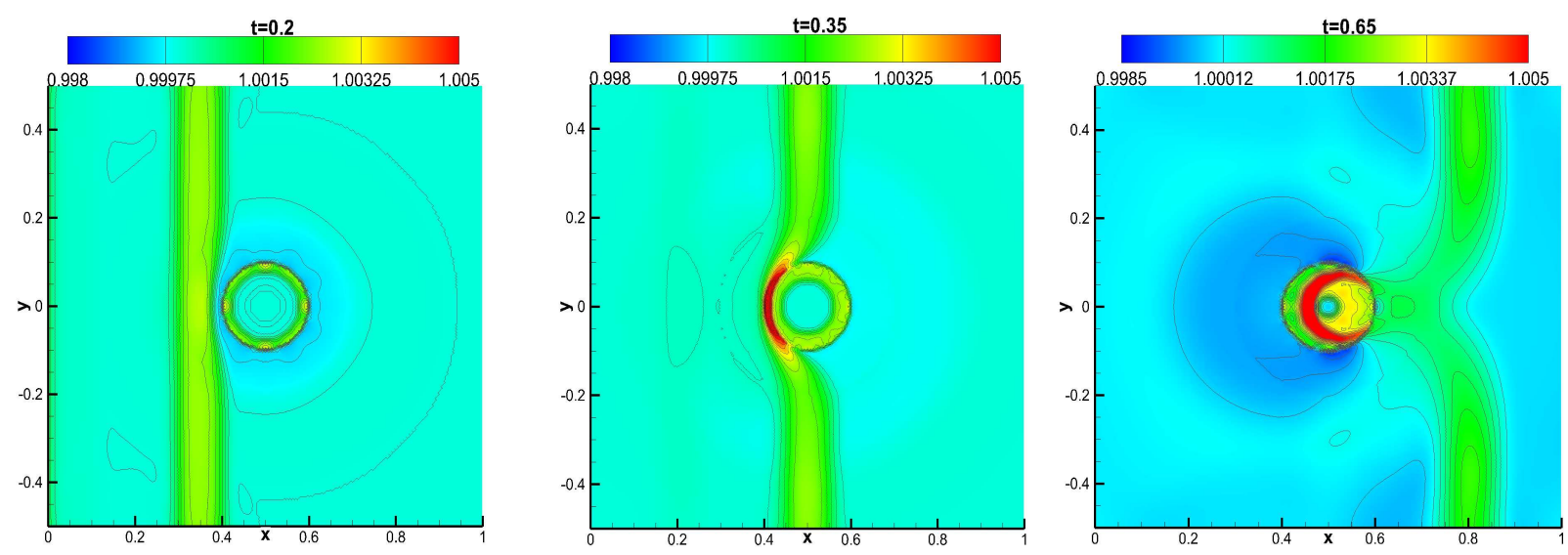

Figure 7: Example 2: Solution $(w)$ computed by the non well-balanced cell-vertex central-upwind scheme. 
by the following bottom topography function:

$$
B(x, y)= \begin{cases}1.1, & r \leq 0.1, \\ 11(0.2-r), & 0.1<r<0.2, \quad r:=\sqrt{x^{2}+y^{2}} \\ 0, & \text { otherwise }\end{cases}
$$

which is located in the center of the computational domain $[-0.5,0.5] \times[-0.5,0.5]$. The initial condition given by

$$
w(x, y, 0)=\left\{\begin{array}{ll}
1+\varepsilon, & -0.4<x<-0.3, \\
\max (1, B(x, y)), & \text { otherwise, }
\end{array} \quad u(x, y, 0) \equiv v(x, y, 0) \equiv 0 .\right.
$$

As in Example 2, the outflow boundary conditions are used in the $x$-direction, while the wall boundary conditions are imposed in the $y$-direction.

The solution computed at times $t=0.35,0.50$ and 0.65 for $\varepsilon=0.01$ using the proposed cell-vertex central-upwind scheme with average cell areas $\left|M_{j}\right|=1.25 \times 10^{-5}$ is shown in Figure 8. The flow around the dry parts of the island is of a special interest. As one can see, the wave bends around the island without any oscillations. On the contrary, the results obtained using a non well-balanced discretization of the source terms contain large artificial waves, which develop completely different solution structure, see Figure 9.

\section{Example 4: Dam Break over Discontinuous Topography}

In this example, we test the ability of the proposed cell-vertex central-upwind scheme to accurately resolve rapidly varying flows. We consider a one-dimensional dam break problem from [44], see also [11], which we solve using the 2-D code with outflow boundary conditions. 

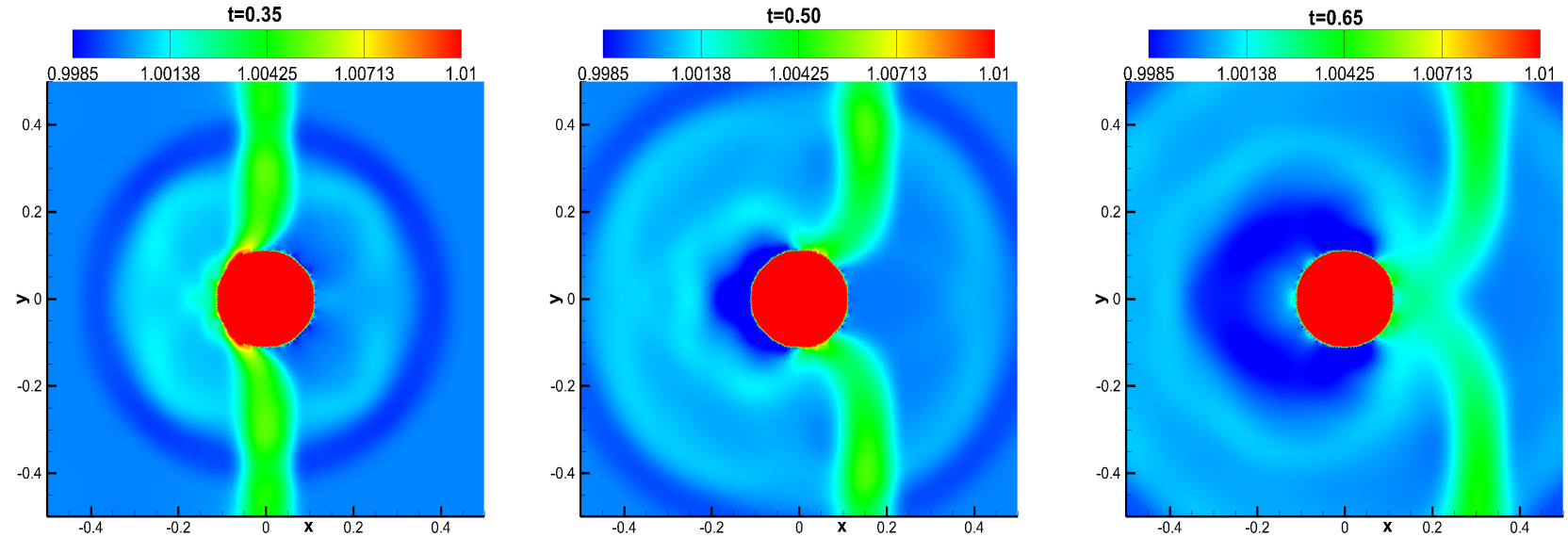

Figure 8: Example 3: Solution $(w)$ computed by the well-balanced cell-vertex central-upwind scheme. The circle in the center of the computational domain represents the part of the bottom that is above the water surface.
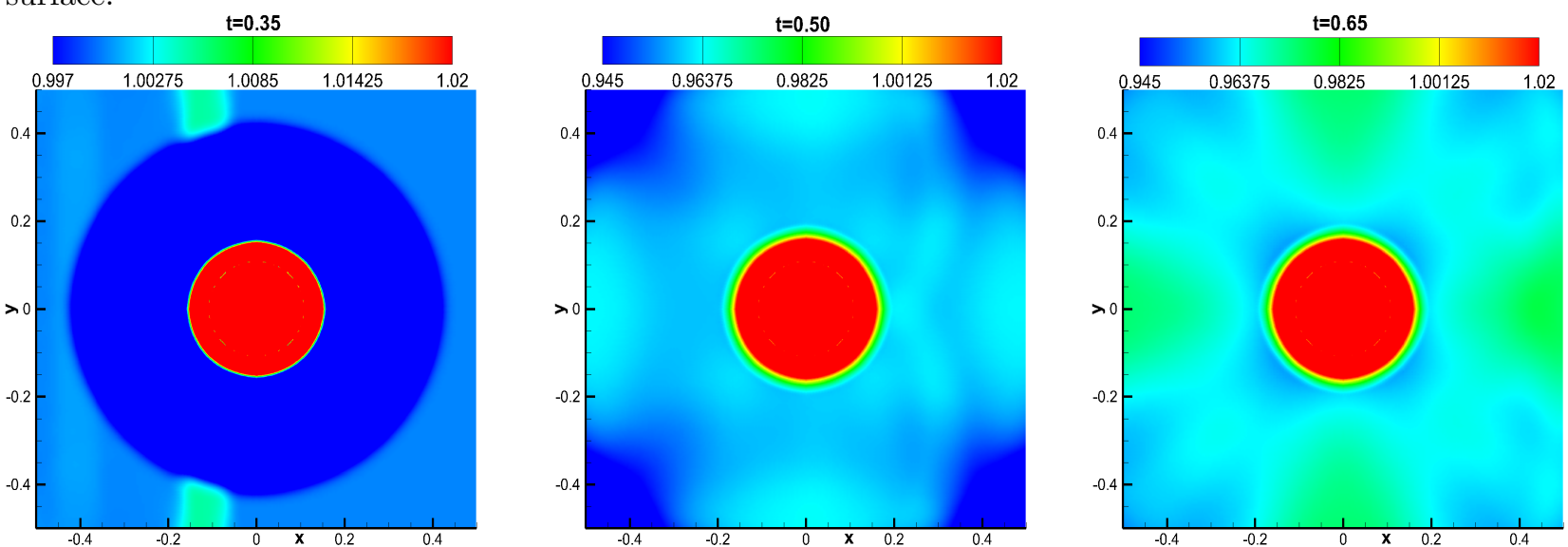

Figure 9: The same as Figure 8, but the solution $(w)$ is computed by the non well-balanced cell-vertex central-upwind scheme.

In this problem, the bottom topography is given by

$$
B(x, y)= \begin{cases}8, & |x-750| \leq 187.5 \\ 0, & \text { otherwise }\end{cases}
$$

and the initial conditions are

$$
w(x, y, 0)=\left\{\begin{array}{ll}
20, & x<750, \\
15, & \text { otherwise },
\end{array} \quad u(x, y, 0) \equiv v(x, y, 0) \equiv 0 .\right.
$$


In Figure 10, we show the solutions computed using the cell-vertex central-upwind scheme at time $t=15$ using the grid shown in Figure 12 (left) with average cell areas $M_{j}=2.30$ and $M_{j}=0.50$. The shock and rarefaction waves reach the discontinuities in the bottom topography at about $t \approx 17$. We then compute the solutions on the same two grids at time $t=55$, at which the developed wave structure is much more complicated than at time $t=15$. The obtained results are shown in Figure 11. The solutions computed by the proposed central-upwind scheme are in a good agreement with the solutions reported in $[11,44]$, they are oscillation-free, and the achieved resolution is very high.
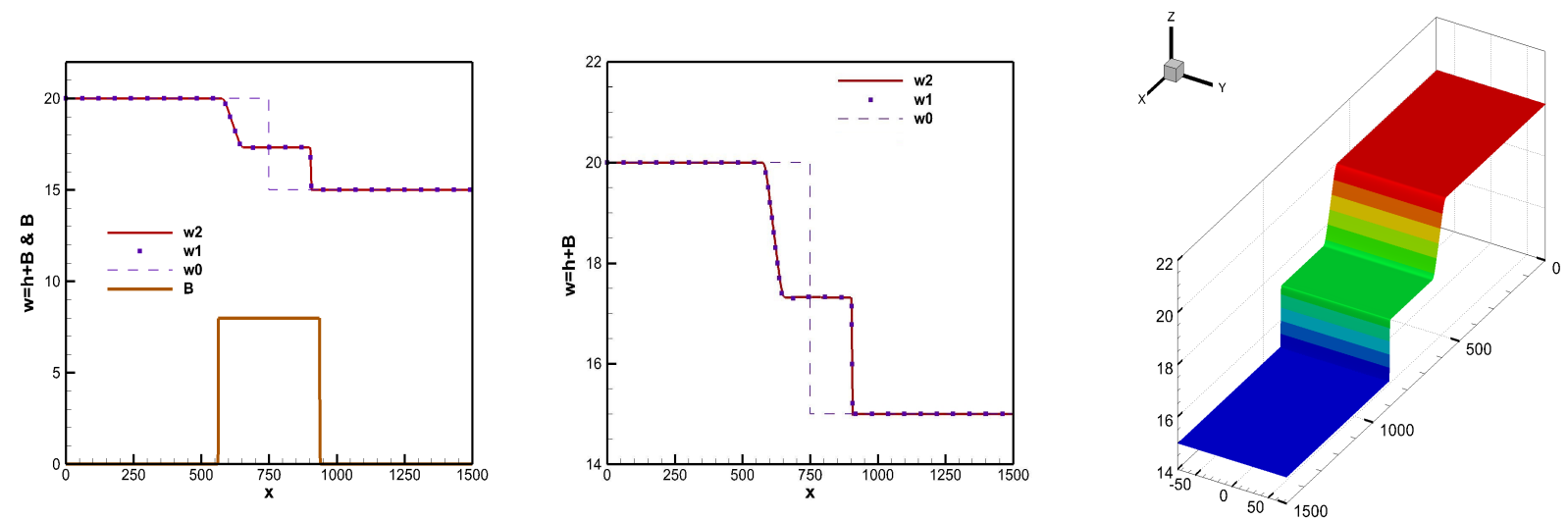

Figure 10: Example 4: Solution at time $t=15$ computed by the proposed cell-vertex central-upwind scheme. In the left and middle graphs, a 1-D slice of the solution along the line $y=0$ is shown. There, $w_{2}$ and $w_{1}$ are the water surface elevations computed using average cell areas $\left|M_{j}\right|=0.50$ and $\left|M_{j}\right|=2.30$, respectively, $w_{0}$ is the initial condition. The bottom topography $B$ is plotted at the left. The $3-\mathrm{D}$ view of the computed water surface is on the right.

\section{Example 5: Thacker's Time-Dependent Solution using a Parabolic Flood Wave}

In the last example, the proposed cell-vertex central-upwind scheme is tested using a time-dependent exact solution proposed by Thacker [43].

For the flat bottom topography $(B \equiv 0)$ and a time reference value $T$, this solution can 

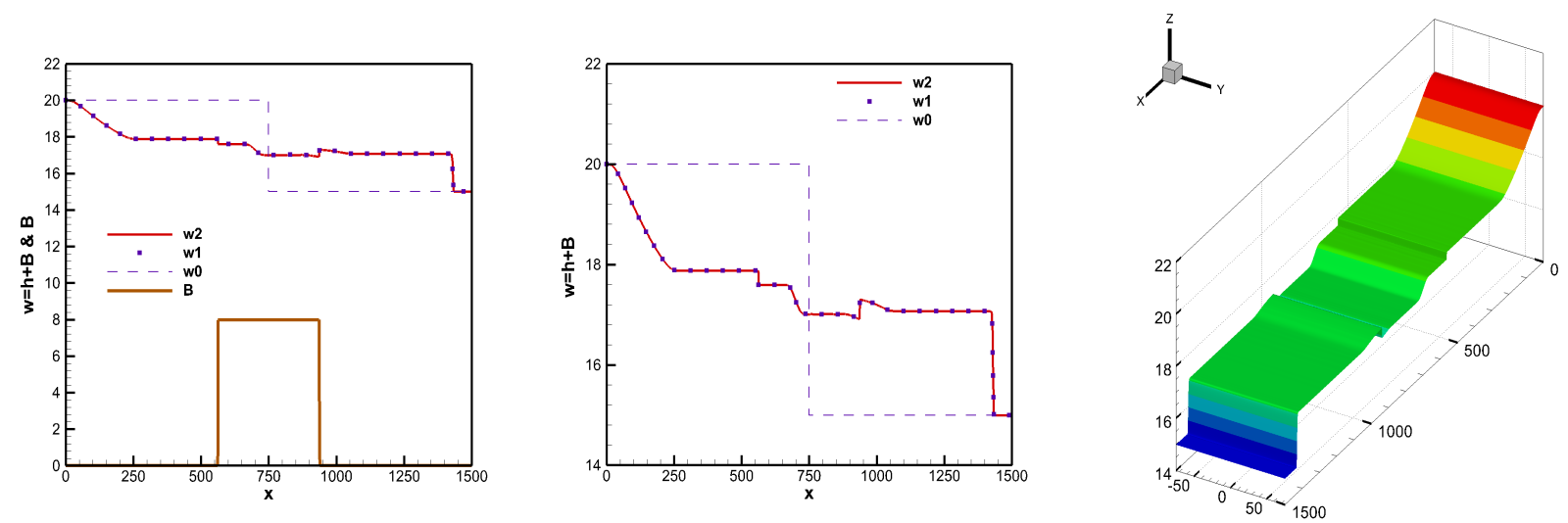

Figure 11: The same as Figure 10, but at a later time $t=55$.
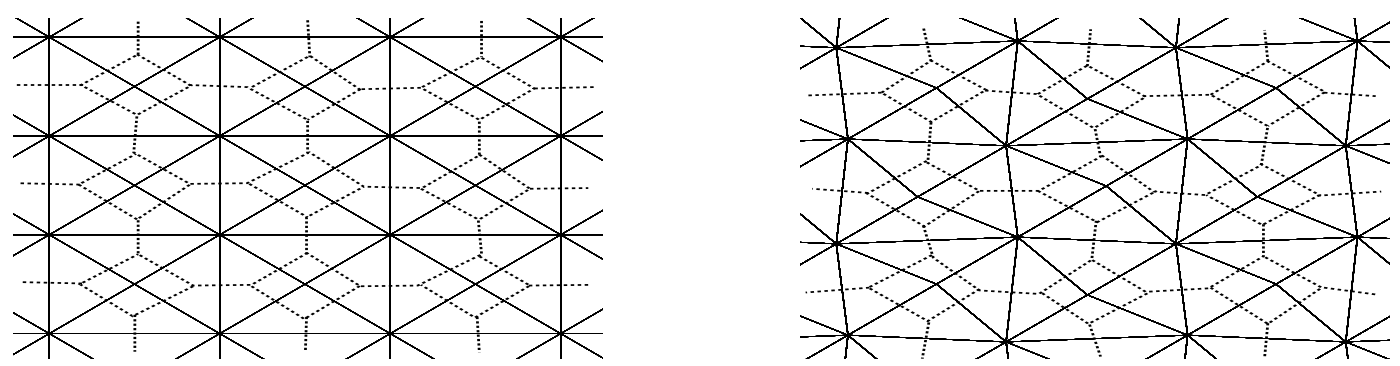

Figure 12: Grids used in Examples 4 (left) and 5 (right). Solid lines represent the primary triangular grids, while the dashed lines show the computational cell-vertex grids.

be written as follows:

$$
\begin{aligned}
& h(x, y, t)=\eta\left[\frac{T^{2}}{t^{2}+T^{2}}-\frac{x^{2}+y^{2}}{R_{0}^{2}}\left(\frac{T^{2}}{t^{2}+T^{2}}\right)^{2}\right], \\
& u(x, y, t)=\frac{x t}{t^{2}+T^{2}}, \quad v(x, y, t)=\frac{y t}{t^{2}+T^{2}},
\end{aligned}
$$

where $\eta$ is the initial height at the peak of the water surface and the parameter $R_{0}$ is the radius of the spreading mound which is given by

$$
R_{0}=T \sqrt{2 g \eta}
$$

This test problem is challenging since for large $t$, the water depth becomes very small. 
We take $\eta=2$ and $R_{0}=9$ and the following initial conditions:

$$
h(x, y, 0)=\eta\left(1-\frac{x^{2}+y^{2}}{R_{0}^{2}}\right), \quad u(x, y, 0) \equiv v(x, y, 0) \equiv 0
$$

on the $[-3,3] \times[-3,3]$ computational domain. The analytical solution (26) is used to calculate the boundary conditions. In Figure 13, we present the solution computed at $t=0.5 T, T$ and $15 T$ using the unstructured mesh-vertex grid shown in Figure 12 (right) with 9941 cells. These results demonstrate that the proposed central-upwind scheme is stable, it preserves the symmetry of the solution and the positivity of the water depth even at large $t=15 T$.

We also test the experimental order of convergence and present the obtained results in Table 1, where the $L^{1}$-errors for $w$ at time $t=T$ and the corresponding convergence rates are shown. Other numerical tests are performed for the spatial order of the proposed method, where a very small time step is used in order to render the temporal errors negligible. Different sizes of the computational cells are used to obtain the errors due to the spatial discretization. Figure 14 shows the evolution of the $L^{1}$-error in the log-log scale, where we observe that for small sizes of the computational cells the spatial convergence rate is approximately equal to 2 . These results confirm the second-order of accuracy of the proposed method.

Table 1: Example 5: Verification of the second-order accuracy of the scheme.

\begin{tabular}{|l|c|c|c|}
\hline Number of cells & Average cell area & $L^{1}$-error & Rate \\
\hline 5101 & $7.06 e-03$ & $2.00 e-03$ & - \\
\hline 9941 & $3.62 e-03$ & $1.34 e-03$ & 1.20 \\
\hline 16381 & $2.20 e-03$ & $8.51 e-04$ & 1.83 \\
\hline 21841 & $1.65 e-03$ & $6.43 e-04$ & 1.95 \\
\hline
\end{tabular}

In order to compare the proposed scheme and the second-order triangular central-upwind 

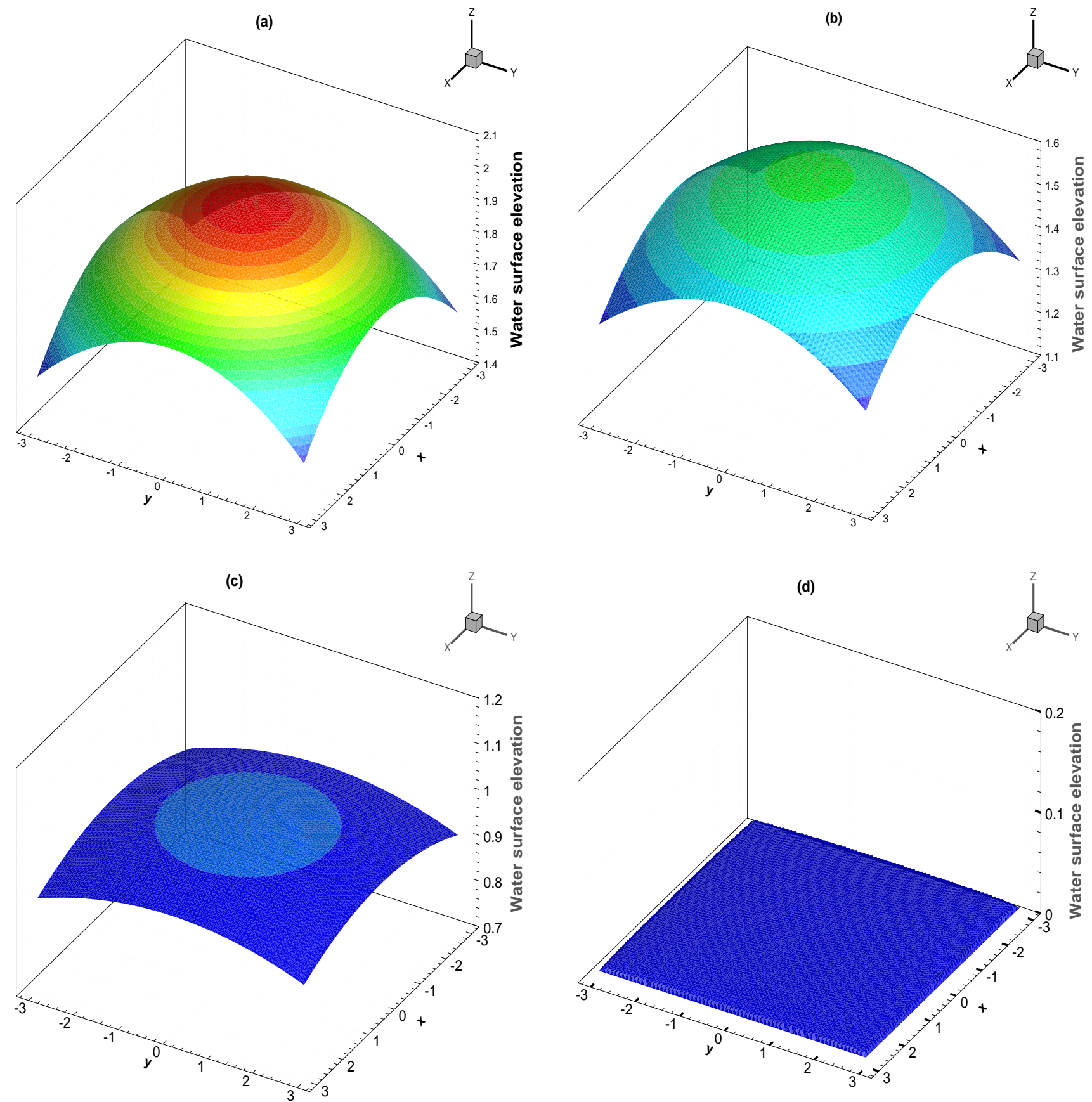

Figure 13: Example 5: Solution $(w)$ computed at (a): $t=0$ (initial condition), (b): $t=0.5 T,(\mathrm{c}): t=T$, (d): $t=15 T$.

scheme in terms of accuracy, the $L^{1}$-error is computed for the solution of parabolic flood wave at time $t=T$ using both schemes. For the CFL number 0.5 , the solution at time $t=T$ 


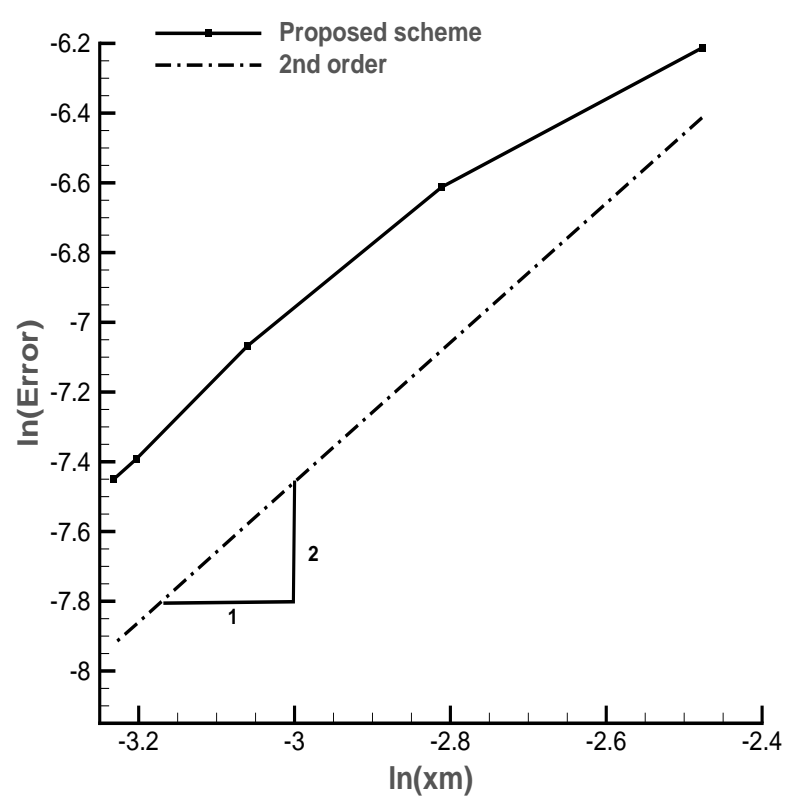

Figure 14: Example 5: Evolution of the $L^{1}$-error in the $\log$-log scale for $w$ at $t=T$ using the proposed scheme with a very small constant time step $\left(\Delta t=10^{-3}\right)$, where xm stands for the square root of the average cell areas.

computed by the proposed scheme using a mesh-vertex computational grid with average cell areas $\left|M_{j}\right|=1.837 \cdot 10^{-3}$ has the $L^{1}$-error $e_{1}=7.140 \cdot 10^{-4}$, while the $L^{1}$-error for the second-order triangular central-upwind scheme using a computational grid with the same average cell areas is $e_{2}=1.922 \cdot 10^{-3}$, which is more than twice larger than $e_{1}$.

\section{Conclusions}

In this paper, we have introduced a new second-order well-balanced positivity preserving central-upwind scheme on unstructured cell-vertex grids for the Saint-Venant system of shallow water equations with variable bottom topography. Cell-vertex finite-volume methods take an advantage of using more cell interfaces. This is important, especially in the case of central-upwind schemes which are developed based on the use of local speeds of propagation at cell interfaces. To develop cell-vertex central-upwind schemes, we have proposed a new piecewise linear approximation of the bottom topography. To ensure the stability of the 
scheme, we have developed a new non-oscillatory piecewise linear reconstruction in which the gradient of each of the conservative variables is computed using a modified minmodtype limiter. The water surface reconstruction has been corrected to guarantee the positivity of the water depth over the entire computational cell. The well-balanced property of the proposed method has been ensured using a special quadrature for the discretization of the cell averages of the source terms due to bottom topography. We have proved the positivity preserving property of the proposed well-balanced cell-vertex central-upwind scheme in the case when the time discretization is carried out using an SSP ODE solver. The third-order SSP Runge-Kutta method has been used in all of our numerical experiments.

The performance of the proposed scheme has been tested on a number of numerical examples. We have used the scheme to compute small perturbations of "lake at rest" steadystate solutions over several different bottom topographies, including a case with almost dry areas and "islands". The scheme has been also validated in the case of a rapidly varying flow over discontinuous bottom topography. In the last numerical example, the accuracy of the proposed scheme has been experimentally tested on a problem for which the exact timedependent solution is available. The obtained numerical results confirm the well-balanced, positivity preserving and non-oscillatory properties as well as the second order of accuracy of the developed cell-vertex central-upwind scheme. Even though an unstructured triangulation was used as a primary grid, an extension to more general polygon-type primary grids can be made in a straightforward manner.

The proposed central-upwind scheme can be extended to multidimensional coupled models, for which the eigenvalues and eigenvectors of the matrix of the system are not known analytically, and thus the solution of the Riemann problem or its numerical approximation may be hard to obtain. In these cases, the use of the proposed Riemann-problem-solver-free central-upwind scheme may be especially advantageous since the estimates on the one-sided local speeds $a_{j k}^{\text {in }}$ and $a_{j k}^{\text {out }}$ may be obtained using the Lagrange theorem [35] as it was done, 
for example, in [14, 29, 32].

\section{Acknowledgments}

This publication was partially made possible by NPRP Grant 4-935-2-354 from the Qatar National Research Fund (a member of the Qatar Foundation) supporting the research of A. Mohammadian and A. Beljadid. The work of A. Kurganov was supported in part by NSF grants DMS-1216957 and DMS-1521009 and ONR grant N00014-12-1-0833.

\section{References}

[1] E. Audusse, F. Bouchut, M.-O. Bristeau, R. Klein, and B. Perthame. A fast and stable well-balanced scheme with hydrostatic reconstruction for shallow water flows. SIAM J. Sci. Comput., 25:2050-2065, 2004.

[2] C. Berthon and F. Foucher. Efficient well-balanced hydrostatic upwind schemes for shallow-water equations. J. Comput. Phys., 231(15):4993-5015, 2012.

[3] J. Blazek. Computational Fluid Dynamics: Principles and Applications. Elsevier, Amsterdam, 2006.

[4] A. Bollermann, G. Chen, A. Kurganov, and S. Noelle. A well-balanced reconstruction of wet/dry fronts for the shallow water equations. J. Sci. Comput., 56(2):267-290, 2013.

[5] P. Brufau and P. García-Navarro. Unsteady free surface flow simulation over complex topography with a multidimensional upwind technique. J. Comput. Phys., 186(2):503-526, 2003.

[6] S. Bryson, Y. Epshteyn, A. Kurganov, and G. Petrova. Well-balanced positivity preserving centralupwind scheme on triangular grids for the Saint-Venant system. M2AN Math. Model. Numer. Anal., 45(3):423-446, 2011.

[7] S. Bryson and D. Levy. Balanced central schemes for the shallow water equations on unstructured grids. SIAM J. Sci. Comput., 27:532-552, 2005.

[8] M. T. Capilla and A. Balaguer-Beser. A new well-balanced non-oscillatory central scheme for the shallow water equations on rectangular meshes. J. Comput. Appl. Math., 252:62-74, 2013.

[9] M. J. Castro, A. Pardo Milanés, and C. Parés. Well-balanced numerical schemes based on a generalized hydrostatic reconstruction technique. Math. Models Methods Appl. Sci., 17(12):2055-2113, 2007. 
[10] L. Cea and M. E. Vázquez-Cendón. Unstructured finite volume discretisation of bed friction and convective flux in solute transport models linked to the shallow water equations. J. Comput. Phys., 231(8):3317-3339, 2012.

[11] Y. Chen, A. Kurganov, M. Lei, and Y. Liu. An adaptive artificial viscosity method for the Saint-Venant system. In Lectures Presented at a Workshop at the Mathematical Research Institute Oberwolfach, Germany, Jan 15-21, 2012; R. Ansorge et al. (Eds.): Recent Developments in the Numerics of Nonlinear Conservation Laws, volume 120 of Notes on Numerical Fluid Mechanics and Multidisciplinary Design, pages 125-141. Springer-Verlag Berlin Heidelberg, 2013.

[12] A. Chertock, S. Cui, A. Kurganov, and T. Wu. Well-balanced positivity preserving central-upwind scheme for the shallow water system with friction terms. Internat. J. Numer. Meth. Fluids, 78:355383,2015 .

[13] A. Chertock, A. Kurganov, and Y. Liu. Central-upwind schemes for the system of shallow water equations with horizontal temperature gradients. Numer. Math., 127(4):595-639, 2014.

[14] A. Chertock, A. Kurganov, Z. Qu, and T. Wu. Three-layer approximation of two-layer shallow water equations. Math. Model. Anal., 18(5):675-693, 2013.

[15] N. Črnjarić-Žic, S. Vuković, and L. Sopta. Improved non-staggered central NT schemes for balance laws with geometrical source terms. Internat. J. Numer. Methods Fluids, 46(8):849-876, 2004.

[16] A.I. Delis, I.K. Nikolos, and M. Kazolea. Performance and comparison of cell-centered and nodecentered unstructured finite volume discretizations for shallow water free surface flows. Arch. Comput. Methods Eng., 18(1):57-118, 2011.

[17] B. Diskin, J.L. Thomas, E.J. Nielsen, H. Nishikawa, and J.A. White. Comparison of node-centered and cell-centered unstructured finite-volume discretizations: Viscous fluxes. AIAA Journal, 48:1326-1338, 2010.

[18] T. Gallouët, J.-M. Hérard, and N. Seguin. Some approximate Godunov schemes to compute shallowwater equations with topography. Comput. \& Fluids, 32(4):479-513, 2003.

[19] S. Gottlieb, D. Ketcheson, and C.-W. Shu. Strong stability preserving Runge-Kutta and multistep time discretizations. World Scientific Publishing Co. Pte. Ltd., Hackensack, NJ, 2011.

[20] S. Gottlieb, C.-W. Shu, and E. Tadmor. Strong stability-preserving high-order time discretization methods. SIAM Rev., 43:89-112, 2001.

[21] D.-A. Ham, S.-C. Kramer, G.-S. Stelling, and J. Pietrzak. The symmetry and stability of unstructured mesh c-grid shallow water models under the influence of coriolis. Ocean Modelling, 16:47-60, 2007. 
[22] G. Hernández-Dueñas and S. Karni. Shallow water flows in channels. J. Sci. Comput., 48(1-3):190-208, 2011.

[23] A. Kurganov and D. Levy. Central-upwind schemes for the Saint-Venant system. Mathematical Modelling and Numerical Analysis, 36:397-425, 2002.

[24] A. Kurganov and J. Miller. Central-upwind scheme for Savage-Hutter type model of submarine landslides and generated tsunami waves. Comput. Methods Appl. Math., 14:177-201, 2014.

[25] A. Kurganov, S. Noelle, and G. Petrova. Semi-discrete central-upwind scheme for hyperbolic conservation laws and Hamilton-Jacobi equations. SIAM J. Sci. Comput., 23:707-740, 2001.

[26] A. Kurganov and G. Petrova. A third-order semi-discrete genuinely multidimensional central scheme for hyperbolic conservation laws and related problems. Numer. Math., 88(4):683-729, 2001.

[27] A. Kurganov and G. Petrova. Central-upwind schemes on triangular grids for hyperbolic systems of conservation laws. Numer. Methods Partial Differential Equations, 21:536-552, 2005.

[28] A. Kurganov and G. Petrova. A second-order well-balanced positivity preserving central-upwind scheme for the Saint-Venant system. Commun. Math. Sci., 5:133-160, 2007.

[29] A. Kurganov and G. Petrova. Central-upwind schemes for two-layer shallow equations. SIAM J. Sci. Comput., 31:1742-1773, 2009.

[30] A. Kurganov and E. Tadmor. New high resolution central schemes for nonlinear conservation laws and convection-diffusion equations. J. Comput. Phys., 160:241-282, 2000.

[31] R. J. LeVeque. Balancing source terms and flux gradients in high-resolution Godunov methods: the quasi-steady wave-propagation algorithm. J. Comput. Phys., 146(1):346-365, 1998.

[32] X. Liu, A. Mohammadian, A. Kurganov, and J. A. Infante Sedano. Well-balanced central scheme for a fully coupled shallow water system modeling flows over erodible bed. J. Comput. Phys., 300:202-218, 2015 .

[33] M. Lukáčová-Medviďová, S. Noelle, and M. Kraft. Well-balanced finite volume evolution Galerkin methods for the shallow water equations. J. Comput. Phys., 221(1):122-147, 2007.

[34] D.J. Mavriplis. Unstructured-mesh discretizations and solvers for computational aerodynamics. AIAA journal, 46(6):1281-1298, 2008.

[35] M. Mignotte and D. Stefanescu. On an estimation of polynomial roots by lagrange. Technical Report 025/2002, pp. 1-17, IRMA Strasbourg, http://hal .archives-ouvertes.fr/hal-00129675/en/, 2002.

[36] K.W. Morton and T. Sonar. Finite volume methods for hyperbolic conservation laws. Acta Numer., 
16:155-238, 2007.

[37] J. Murillo and P. García-Navarro. Weak solutions for partial differential equations with source terms: application to the shallow water equations. J. Comput. Phys., 229(11):4327-4368, 2010.

[38] H. Nessyahu and E. Tadmor. Nonoscillatory central differencing for hyperbolic conservation laws. $J$. Comput. Phys., 87(2):408-463, 1990.

[39] I.K. Nikolos and A.I. Delis. An unstructured node-centered finite volume scheme for shallow water flows with wet-dry fronts over complex topography. Comput. Methods Appl. Mech. Engrg., 198(4748):3723-3750, 2009.

[40] G. Russo. Central schemes and systems of balance laws. In Hyperbolic Partial Differential Equations, Theory, Numerics and Applications, pages 59-114. A. Meister and I. Struckmeier, Vieweg, Wiesbaden (D), Braunschweig, 2002.

[41] G. Russo. Central schemes for conservation laws with application to shallow water equations. pages $225-246,2005$.

[42] C-W. Shu and S. Osher. Efficient implementation of essentially non-oscillatory shock-capturing schemes. Int. J. Comput. Fluid Dyn., 77:439-71, 1988.

[43] W.C. Thacker. Some exact solutions to the nonlinear shallow-water wave equations. J. Fluid Mech, 107:499-508, 1981

[44] S. Vukovic and L. Sopta. ENO and WENO schemes with the exact conservation property for onedimensional shallow water equations. J. Comput. Phys., 179(2):593-621, 2002.

[45] S. Yang, A. Kurganov, and Y. Liu. Well-balanced central schemes on overlapping cells with constant subtraction techniques for the Saint-Venant shallow water system. J. Sci. Comput., 63(3):678-698, 2015. 